\title{
Phosphor-Free Solid State Light Sources
}

\author{
Final Technical Report
}

Report Period: 10/1/03 - 3/31/07

Principal Authors:

Jeff E. Nause: Cermet, Inc.

Ian Ferguson: Georgia Institute of Technology

Alan Doolittle: Georgia Institute of Technology

Date: September 18, 2007

DOE Award Number: DE-FG26-03NT41942

Name and Address of Submitting Organization:

\author{
Cermet, Inc. \\ 1019 Collier Road \\ Building C \\ Atlanta, GA 30318 \\ Georgia Institute of Technology \\ 400 North Avenue \\ Atlanta, GA 30318
}

\begin{abstract}
Disclaimer
This report was prepared as an account of work sponsored by an agency of the United States Govemment. Neither the United States Govemment nor any agency thereof, nor any of their employees, makes any warranty, express or implied, or assumes any legal liability or responsibility for the accuracy, completeness, or usefulness of any information, apparatus, product, or process disclosed, or represents that its use would not infringe privately owned rights. Reference herein to any specific commencial product, process, or service by trade name, trademark, manufacturer, or otherwise does not necessarily constitute or imply its endorsement, recommendation, or favoring by the United States Govemment or any agency thereof. The views and opinions of authors expressed herein do not necessarily state or reflect those of the United States Government or any agency thereof.
\end{abstract}




\begin{abstract}
The objective of this work was to demonstrate a light emitting diode that emitted white light without the aid of a phosphor. The device was based on the combination of a nitride LED and a fluorescing $\mathrm{ZnO}$ substrate. The early portion of the work focused on the growth of $\mathrm{ZnO}$ in undoped and doped form. The doped $\mathrm{ZnO}$ was successfully engineered to emit light at specific wavelengths by incorporating various dopants into the crystalline lattice. Thereafter, the focus of the work shifted to the epitaxial growth of nitride structures on $\mathrm{ZnO}$. Initially, the epitaxy was accomplished with molecular beam epitaxy (MBE). Later in the program, metallorganic chemical vapor deposition (MOCVD) was successfully used to grow nitrides on $\mathrm{ZnO}$. By combining the characteristics of the doped ZnO substrate with epitaxially grown nitride LED structures, a phosphor-free white light emitting diode was successfully demonstrated and characterized.
\end{abstract}


TABLE OF CONTENTS

ABSTRACT

INTRODUCTION

RESULTS AND DISCUSSION

CONCLUSIONS
Page 2

Page 4

Page 4

Page 33 


\section{INTRODUCTION}

The overall project objective is to demonstrate a phosphor-free white LED technology. One goal for this objective is to produce a high quality, doped $\mathrm{ZnO}$ substrate as both the lattice-matched substrate for a nitride LED and an emission source for white light generation. An additional goal is the production of a low defect density, high power nitride LED on doped $\mathrm{ZnO}$, which will serve as the optical pump for the doped substrate. The work will be performed both at Cermet and at the Georgia Institute of Technology, with Cemet focusing on the bulk development and Georgia Tech focusing on nitride epitaxy development, which will demand an efficient interaction between the staff at Cermet and Drs. Ferguson and Doolittle at Georgia Tech.

Project Milestones:

\begin{tabular}{|l|l|}
\hline Attribute & End of Project \\
\hline Production of doped ZnO substrates & $\begin{array}{l}\text { Size: } \\
\text { 50mm diameter; } \\
\text { Crystal Quality: }<100 \text { arcsec; } \\
\text { Optical Quality: }>\mathbf{8 0 \%} \text { optical } \\
\text { transparency (undoped) }\end{array}$ \\
\hline $\begin{array}{l}\text { Production of lattice-matched nitride } \\
\text { emitters on ZnO }\end{array}$ & $\begin{array}{l}\text { Pilot production of lattice-matched } \\
\text { InGaN on ZnO in a commercial } \\
\text { reactor }\end{array}$ \\
\hline $\begin{array}{l}\text { Production of phosphor-free white LED } \\
\text { (white light from lattice-matched } \\
\text { nitride/ZnO device) }\end{array}$ & $\begin{array}{l}\text { 200\% increase in light output from } \\
\text { Year 2 results }\end{array}$ \\
\hline
\end{tabular}

\section{RESULTS AND DISCUSSION \\ Quick Results Overview}

Cemmet provided $\mathrm{ZnO}$ substrates using its patented crystal growth process and prepared production grade substrates using mechanical and chemi-mechanical polishing processes, which eliminate common defects and provide excellent surface finish, crystallinity, and orientation.

Georgia Tech focused on lattice-matched InGaN epitaxy on $\mathrm{ZnO}$ substrates. Dr. Ferguson developed the growth processes for lattice-matched epitaxial InGaN on bulk ZnO using MOCVD. Some growths were also attempted by molecular beam epitaxy (MBE). This approach has achieved the following:

- Growth of high quality, high indium-containing InGaN alloys. Incorporation of high In compositions were achieved by MOCVD with lower growth temperatures $<800^{\circ} \mathrm{C}$. The process was hydrogen-free with only nitrogen as the carrier gas.

- Controlled In fluctuations during growth to obtain $\operatorname{In}_{0.18} \mathrm{Ga}_{0.82} \mathrm{~N}$ alloy. Indium segregation can occur during InGaN growth and control of this mechanism has proved important in optimizing efficient emitters on highly mismatched substrates 
such as sapphire. In this project, phase separation has occurred at high In concentration on sapphire but not on $\mathrm{ZnO}$. This can be due to control over growth conditions, substrate choice, etc., such that they would be optimum for near lattice-matched conditions.

- Characterization of nitride films grown on bulk $\mathrm{ZnO}$. The results of the characterization studies served as a process control for the direction of future epitaxy experiments. Characterization focused on verification of alloy composition, defect reduction, and strain.

Origin of the problems faced:

$\mathrm{ZnO}$ as a Substrate for GaN Growth Progress in the growth of $\mathrm{GaN}$ materials has enabled the production of many new optoelectronic devices that operate at shorter wavelengths than previous materials allowed. The main challenge in fabricating these devices has been in growing high quality GaN and GaN-based alloys on non-native

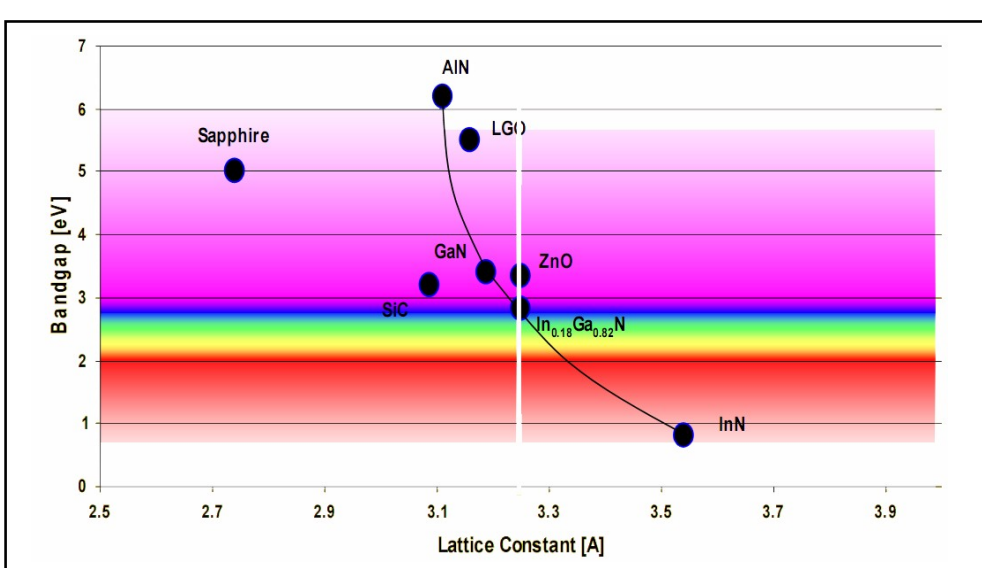

Figure 1. Lattice constants and bandgap energies of III-N alloys and various substrate materials.

substrate materials, such as sapphire $\left(\mathrm{Al}_{2} \mathrm{O}_{3}\right)$ and $\mathrm{SiC}$, which have large mismatches in lattice constant and themal expansion coefficient. An altemate approach is to use heteroepitaxial growth techniques on a lattice-matched substrate, as this can lead to a low epitaxial strain and a lower critical thickness before the appearance of misfit dislocations. However, even the identification of lattice-matched substrates in GaN is problematic. Instead of being the cubic zincblende structure as is common in most III-V semiconductors, the equilibrium phase of GaN is the hexagonal wurtzite phase. Thus, there are fewer substrates available simply because of the lower relative number of hexagonal compounds to cubic compounds. Also, due to GaN being a refractory material with a melting temperature greater than $1800 \mathrm{~K}$, it is very difficult to produce single crystals of GaN by the standard single crystal growth techniques. Current growth methods for GaN substrates are not readily available at large sizes.

$\mathrm{ZnO}$ offers many advantages over these previous materials due to its many structural similarities to GaN. Both are wurtzite crystal structures with identical stacking order, closely matched lattice constants, and similar themal expansion coefficients. In addition, $\mathrm{ZnO}$ is perfectly lattice matched to an $18 \%$ InGaN alloy, a material necessary for green emission devices, see Figure 1. Finally, because $\mathrm{ZnO}$ has high electrical conductivity, devices grown on $\mathrm{ZnO}$ can use back contacts, which can improve current spreading. 
Because of its many inherent advantages, $\mathrm{ZnO}$ has been investigated for use as a buffer layer, and more recently as a bulk substrate material for the growth of GaN. There have been several reports of successful growth of $\mathrm{GaN}$ on $\mathrm{ZnO}$ via molecular beam epitaxy (MBE) and pulsed layer deposition (PLD). Early MBE results showed that the polarity of GaN films grown on $c$-plane $\mathrm{ZnO}$ is somewhat dependant on the polarity of the substrate, with GaN grown on O-face $\mathrm{ZnO}$ exhibiting better crystal quality $[1,2]$, though more recent reports have suggested that proper surface preparation and control of growth parameters can yield good quality GaN films on the $\mathrm{Zn}$ face also [3]. GaN films grown on bulk $\mathrm{ZnO}$ also showed reduced strain and reduced density of defects compared to films grown on sapphire, Figure 2.

The presence of a built in electrostatic field due to the polarity of $c$-plane layers has been identified as having an adverse effect on GaN device performance [4]. The proposed solution to this problem is to grow GaN on nonpolar planes, such as the $a$ - and $m$ planes. However, relatively little altemative plane GaN growth has been done on bulk $\mathrm{ZnO}$, probably because high quality $\mathrm{ZnO}$ substrates have only recently become available and economical. One group has reported successful MBE growth of epitaxial GaN on $a$-plane $\mathrm{ZnO}$, which they achieved by incorporating a latticematched InGaN buffer layer [5].

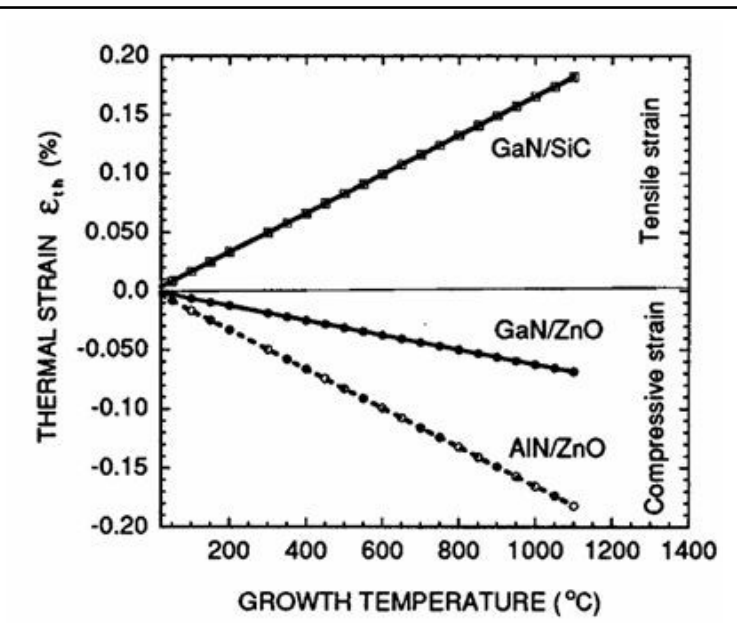

Figure 2. Themal induced strain calculations for $\mathrm{GaN} / \mathrm{ZnO}$, $\mathrm{AIN} / \mathrm{ZnO}$, and GaN/SiC [1].

\section{Results:}

\section{Production of doped $\mathrm{ZnO}$ Substrates}

\section{Melt Growth Process}

$\mathrm{ZnO}$ is a relatively difficult material to melt and grow single crystals from the liquid phase due to its high melting point $(-2250 \mathrm{~K})$ coupled with its proclivity to dissociate (beginning at $\sim 980 \mathrm{~K}$ ). The crystal growth apparatus utilizes a modified Bridgeman growth technique including a pressure vessel that contains pressurized oxygen, Figure 3 (1). The apparatus also includes a cooling unit (2) that is situated in the pressure vessel. The cooling unit receives a coolant flow from outside of the vessel (3) and has cooled surfaces that define an enclosure, which receives the $\mathrm{ZnO}$ with proper dopant concentration $\left(10^{15}-10^{20}\right.$ atoms $/$ cc).

The apparatus further includes an inductive heating element (4) situated in the vessel, which is coupled to receive radiofrequency (RF) power extemally to the vessel (5). The element heats the interior portion of the doped $\mathrm{ZnO}$ to form a molten interior portion contained by a relatively cool, exterior solid-phase portion of the doped $\mathrm{ZnO}$ that is closer relative to the molten interior, to the cooled 
surfaces of the cooling unit. Due to the pressure exerted by the gas contained in the vessel, the liquid interior of the doped $\mathrm{ZnO}$ becomes congruently melting to prevent its decomposition. The cooling unit is then lowered (6) through the element to produce crystal nucleation at the base of the cooling unit and preferential crystal growth through the distance traveled.

In addition to RF power, the heating element receives a coolant flow (7) from a feedthrough that extends through a wall of the pressure vessel. In proximity to the vessel wall, the feedthrough has two coaxial conductors (8) to improve the electric power transfer to the heating element and to reduce heating of the extemal

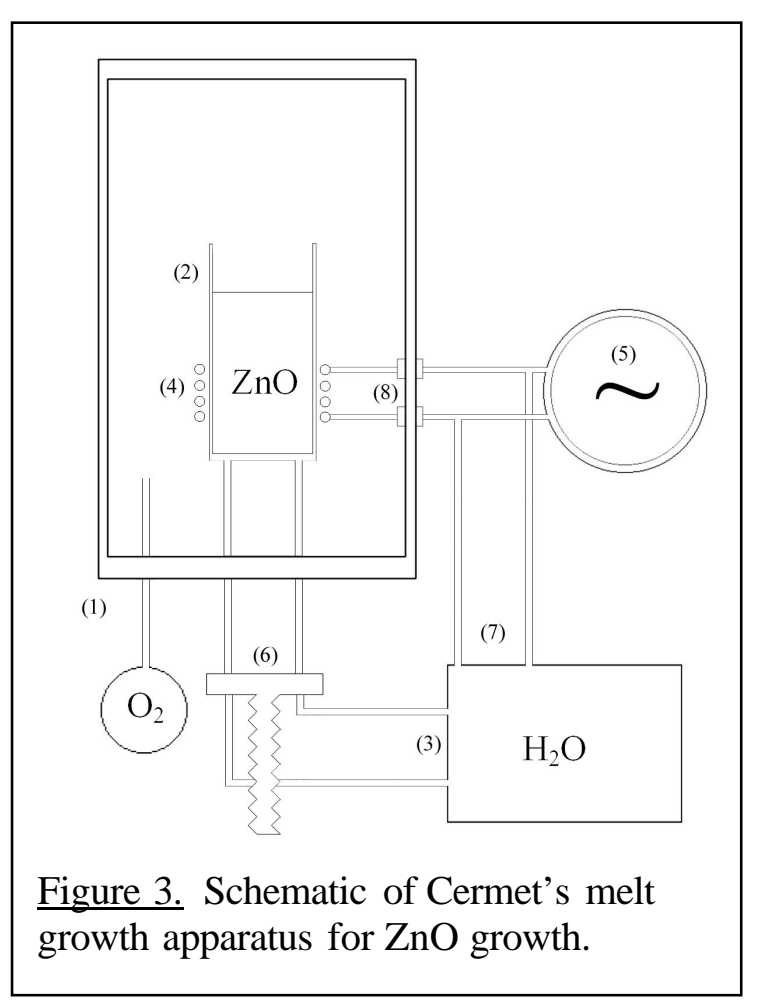
surfaces of the vessel. The two conductors of the feedthrough are cylindrical in shape, and define two channels for channeling a coolant flow to and from, respectively, the heating element.

\section{$\underline{\text { ZnO Doping }}$}

It has been shown that to achieve room temperature emission from rare earths, it is necessary to use a wide bandgap host. ${ }^{\mathrm{i}}$. Furthermore, it has been shown that oxygen incorporation is critical in to produce the characteristic emission of some rare earth ions ${ }^{\mathrm{ii}}$. $\mathrm{ZnO}$ meets both of these requirements. Candidate rare earth dopants have been identified from literature sources to produce the desired characteristic emissions as follows: blue ( $\mathrm{Tm}$ and $\mathrm{Ce}$ ), green ( $\mathrm{Er}, \mathrm{Tb}, \mathrm{Ce}$, and $\mathrm{Ho}$ ), red ( $\mathrm{Pr}, \mathrm{Eu}$, and $\mathrm{Sm})$. Table I lists the various dopants incorporated into the $\mathrm{ZnO}$ crystal during growth. 
Table 1. Doped $\mathrm{ZnO}$ crystals grown.

\begin{tabular}{|l|l|}
\hline $11-23$ & $10^{19} \mathrm{Ho}$ \\
\hline $11-30$ & $10^{19} \mathrm{Er}$ \\
\hline $11-45$ & $10^{19} \mathrm{Li}$ \\
\hline $11-50$ & $10^{19} \mathrm{Gd}$ \\
\hline $11-53$ & $10^{19}(\mathrm{Er}+\mathrm{Li})$ \\
\hline $11-54$ & $10^{19}(\mathrm{Ce}+\mathrm{Li})$ \\
\hline $11-55$ & $10^{19} \mathrm{Tm}$ \\
\hline $11-56$ & $10^{19} \mathrm{La}$ \\
\hline $11-58$ & $10^{20} \mathrm{Er}$ \\
\hline $11-59$ & $10^{19} \mathrm{~Tb}$ \\
\hline $11-60$ & $10^{19} \mathrm{Sc}$ \\
\hline $11-61$ & $10^{18} \mathrm{Er}$ \\
\hline $11-63$ & $10^{19}(\mathrm{Er}+\mathrm{Tm})$ \\
\hline $11-66$ & $10^{19} \mathrm{Er}\left(6^{\prime \prime}\right)$ \\
\hline
\end{tabular}

Transmission data has been measured using a Perkin-Elmer Lambda 10 spectrometer. Excellent transmission in the visible range can be seen for several samples, Figure 4.

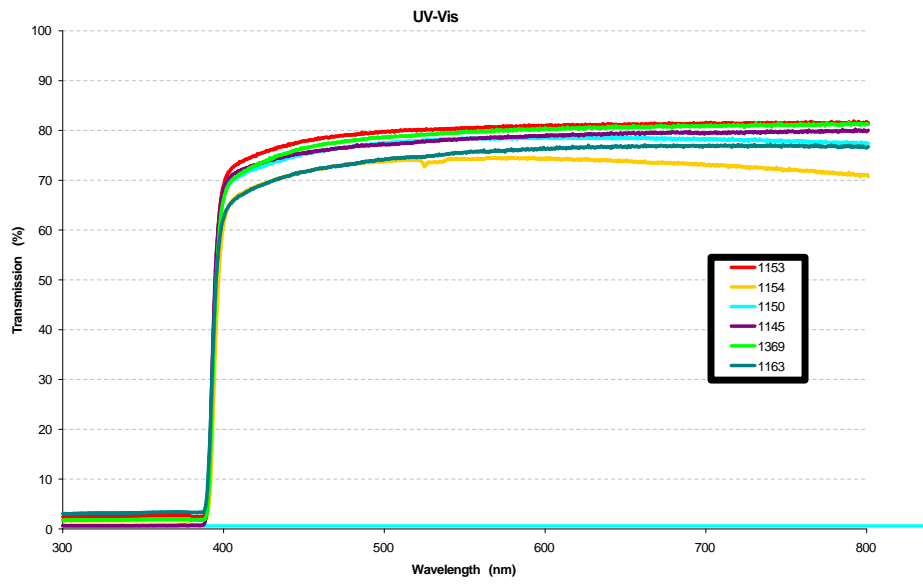

Figure 4. UV-Visible transmission of rare earth doped bulk $\mathrm{ZnO}$ wafers. 
Crystal Size

The cold crucible technique of single crystal melt growth has one key advantage over hot wall crucible techniques because impurities are isolated from incorporating into grown single crystal material because a crust (called a skull) of the grown material encapsulates the melt pool. However,

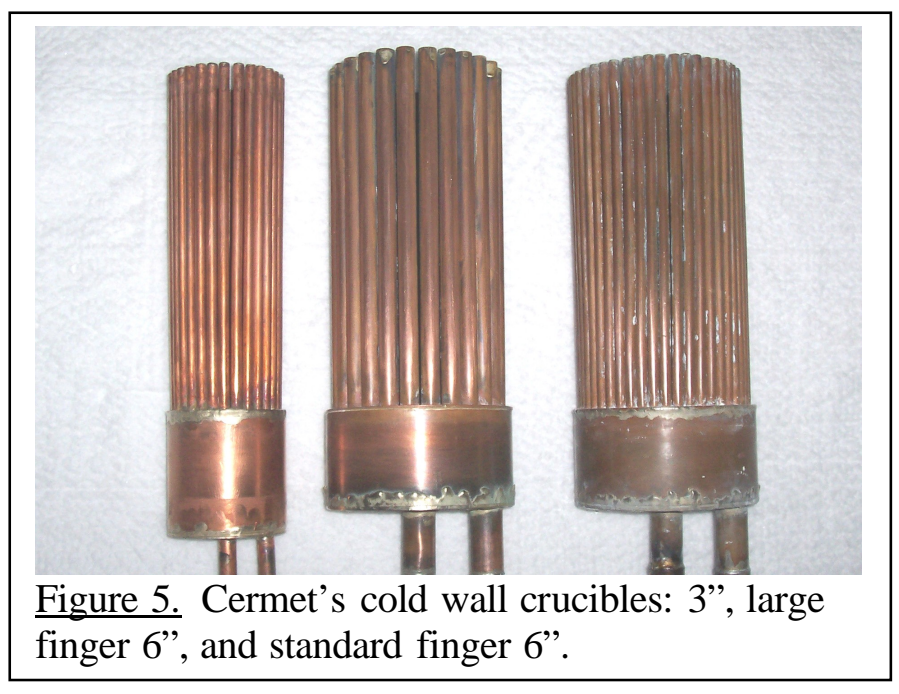

thermal management becomes a resultant key complication due to the high thermal gradients found throughout the volume of the melt. The design of the crucible is a fundamental way to address these themal issues. Figure 5 shows a 3 " crucible and two 6" diameter crucibles.

The fundamental mechanism by which the $\mathrm{ZnO}$ is actually melted is by radio frequency (RF) power creating eddy currents in the $\mathrm{ZnO}$ charge, thereby creating runaway joule heating. This joule heating is primarily confined to an annular region toward the perimeter of the charge of a certain thickness. The remainder of the melt relies on two mechanisms: 1) heat conduction through the $\mathrm{ZnO}$ from this annular region into the center of the charge to produce a complete melt and 2) the RF power, which doesn't heat as effectively toward the center but will still contribute to heating. Since the heat conducted out of the melt will be primarily through the base of the crucible, it is important to have an idea of how much actual power is being consumed through this base.

Most undoped growths were initially performed in 6" crucibles. Scaling the growths to larger crucibles creates a unique challenge since there exists a competition between the heat flow through the $\mathrm{ZnO}$ charge and the heat flow through the base of the crucible. Since thermal conductivities of the crucible materials and the $\mathrm{ZnO}$ are constant, the heat conduction mechanism can be condensed into an area difference to scale

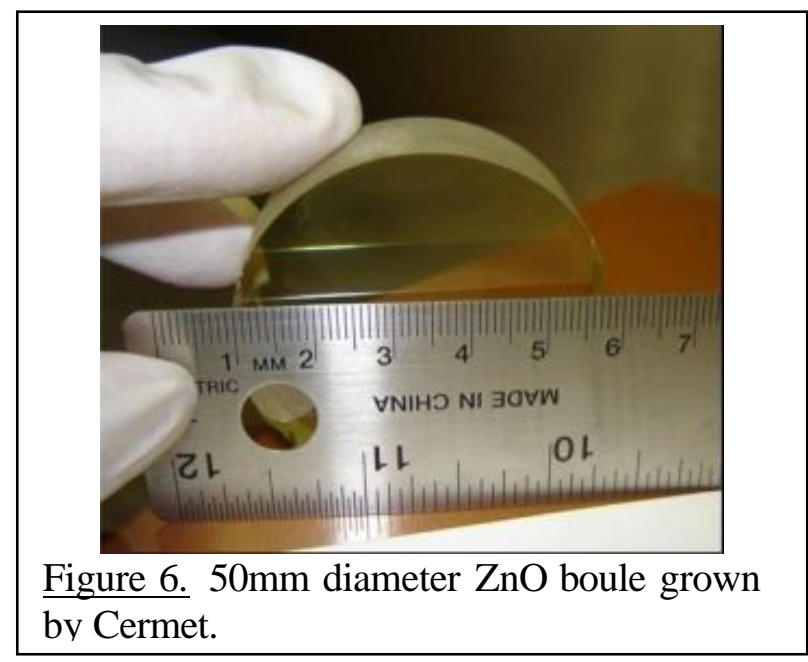
the process. Once both the installation of a higher power RF generator as well as 
improvements to the water cooling system were made, growths in 8" crucibles were conducted. These growths yielded larger, more usable volumes of $\mathrm{ZnO}$ crystals. An example of a 50mm bulk $\mathrm{ZnO}$ crystal boule can be seen in Figure 6 .

\section{Crystal Quality}

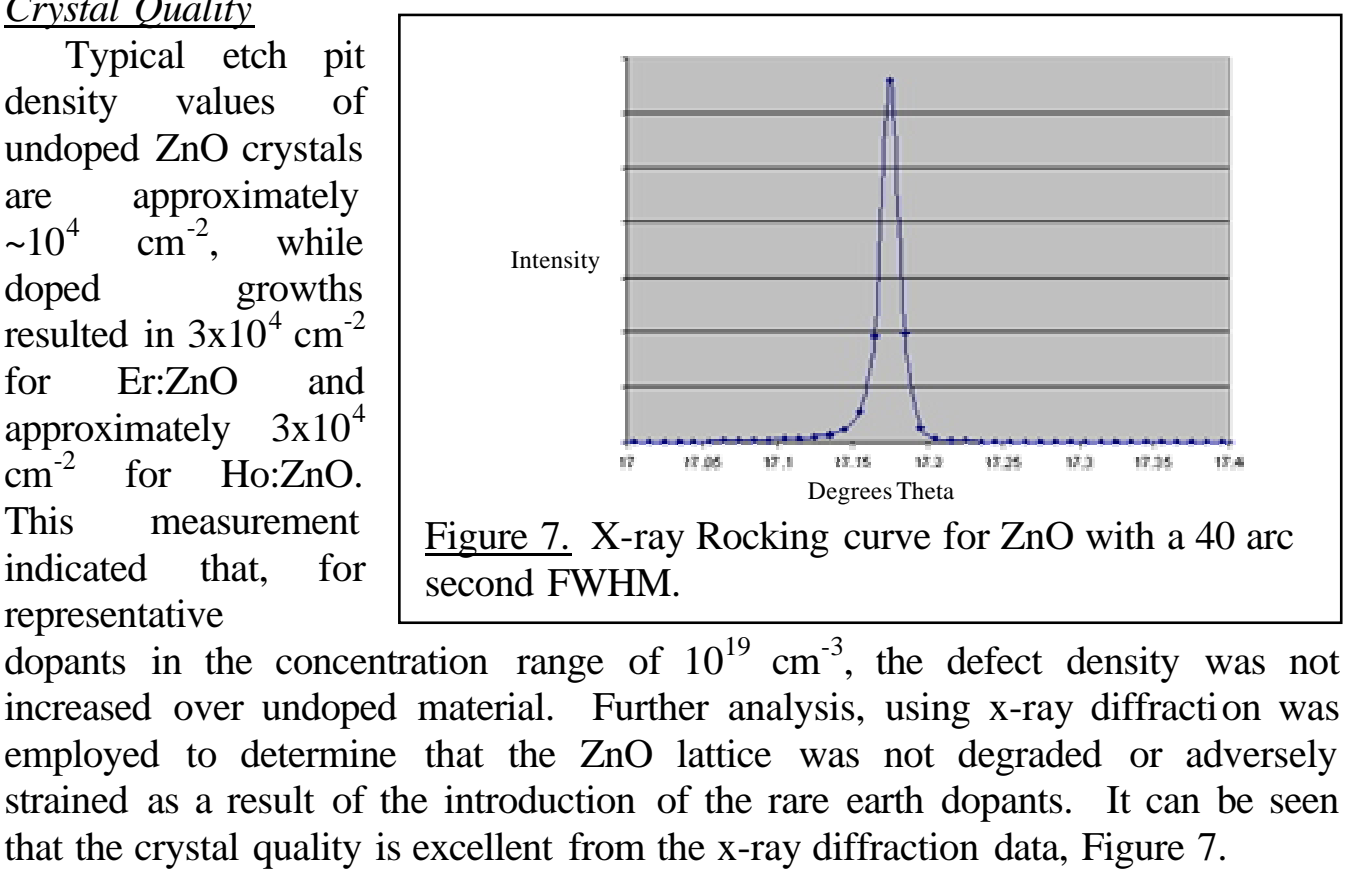

\section{Metalorganic Chemical Vapor Deposition}

The GaN growth system at Georgia Tech used in this study is based on a commencial system but has been specially modified for this work. A photograph of the growth tool shows the reactor on the left and the load lock used to transfer the wafer carrier into the reactor on the right hand side, Figure 8. The system is designed to accommodate growth temperatures ranging up to over $1200^{\circ} \mathrm{C}$. Moreover, the system has been designed to be able to rapidly switch between two

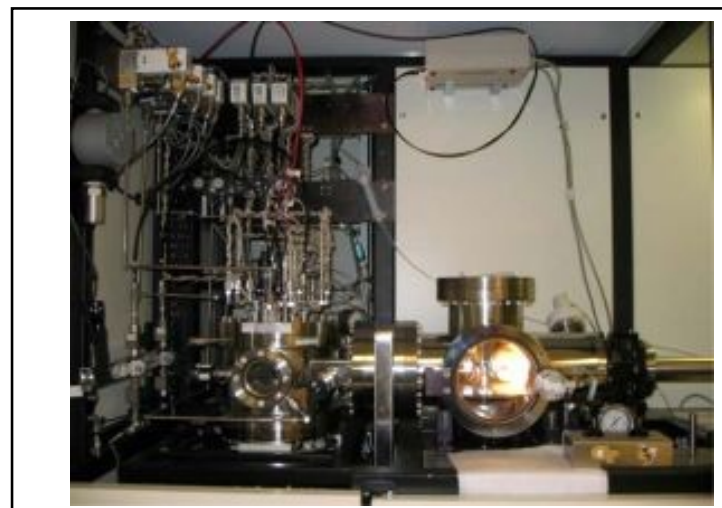

Figure 8. MOCVD tool used in this study.

carrier gases, such that depending on the environment and chemistry, either nitrogen or hydrogen can be used as the carrier gas. Another potentially significant modification to this reactor system is shown in Figure 9. In this schematic, a diagram of the dual injector blocks used in the system shows the ability to flow precursors in separate lines. Very little is known about the use of some MO sources outside of the traditionally used sources during MOCVD growth, and it is essential to avoid gas phase prereactions or precipitation in the lines if high quality material is going to be grown. 


\section{Summary of Challenges}

There are a number of obstacles that need to be addressed for the MOCVD growth of GaN based materials on $\mathrm{ZnO}$. These include $\mathrm{Zn} / \mathrm{O}$ diffusion from the substrate and $\mathrm{H}_{2}$ back etching during high growth temperature. Commencial MOCVD usually grow over $1000^{\circ} \mathrm{C}$ for GaN on sapphire substrates, which makes it difficult for the MOCVD growth of GaN and InGaN on $\mathrm{ZnO}$ substrates. Below are some key points to describe the challenges of GaN based materials grown on

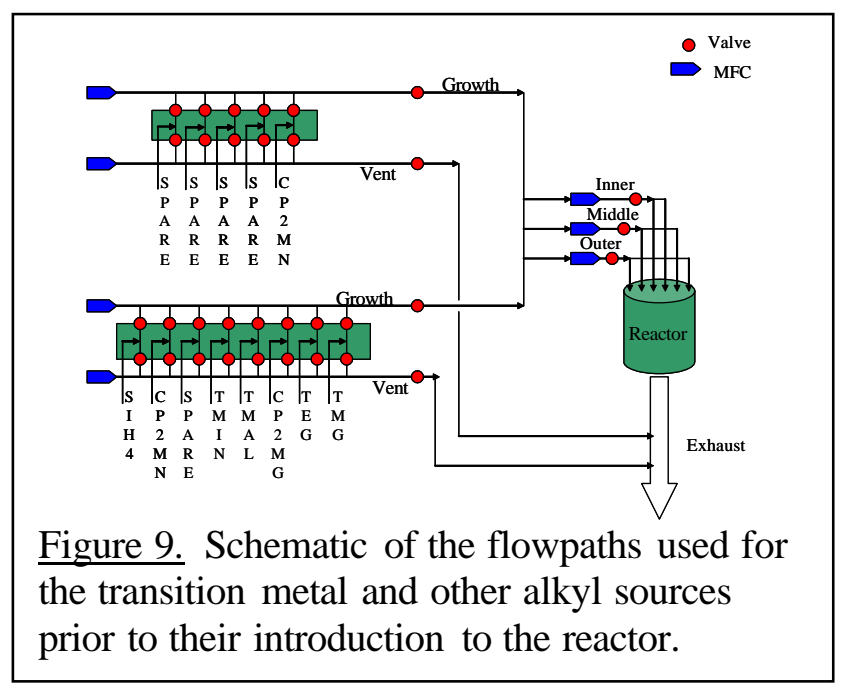
ZnO substrate.

Challenge $1-\mathrm{H}_{2}$ back etching of $\mathrm{ZnO}$ surface: $\mathrm{H}_{2}$ is widely used as a carrier gas for GaN based epilayers grown on sapphire during MOCVD growth. In addition, $\mathrm{NH}_{3}$ contributes $\mathrm{H}_{2}$ during growth especially at high temperature.

Solution $-\mathrm{H}_{2}$ free growth: $\mathrm{A}_{2}$ free growth process has been developed by using $\mathrm{N}_{2}$ as a carrier gas for the entire growth process. In addition, in order to avoid $\mathbf{H}_{2}$ contribution from $\mathrm{NH}_{3}$, DMHy and TBHy were investigated as a protective gas preventing GaN from decomposition at high temperature annealing. However, lowtemperature decomposition $\left(100 \%\right.$ below $\left.800^{\circ} \mathrm{C}\right)$ of $\mathrm{DMHy}$ and $\mathrm{TBHy}$ were found to cause high levels of carbon contamination making high quality GaN growth impossible. Therefore, $\mathrm{NH}_{3}$ was still used during growth.

Challenge 2 - Cracking and peeling off from GaN surface during high temperature annealing: Possible origins for the cracking are phase transformation of the $\mathrm{GaN}-\mathrm{ZnO}$ interface and re-crystallization during high temperature ramping (above $900^{\circ} \mathrm{C}$ ) during annealing. This approach leads to a concentration of stress in the grain boundaries. As a result, $\mathrm{NH}_{3}$ can diffuse through the cracks forming voids undemeath the cracks, degrading the film quality and making the high temperature epitaxy impossible. This mechanism, if it continues to advance, will cause peeling off of the GaN surface, where the possible origin of the peeling off and etch pits are the pinhole formations in the LT-GaN multi-buffer layers.

Solution -Multi-buffer layer: A two-step GaN buffer layer was used to resolve the cracking surface. The $2^{\text {nd }}$ GaN buffer layer covers the pinholes from the 1 st GaN buffer. This ensures the surface is completely and uniformly covered by GaN before high-temperature growth. In a further work, AIN was used in place of the first GaN buffer to help with surface peeling off due to aluminum's low surface mobility and high surface coverage. AlN provides a more thorough surface coverage on $\mathrm{ZnO}$ allowing for lower surface pinholes during the high temperature crystallization step. 
It has a stronger bond than Ga forming a higher stable interfacial layer for high temperature growth.

Challenge 3 - High temperature GaN growth induces decomposition of $\mathrm{Zn}$ and $\mathrm{O}$ from $\mathrm{ZnO}$ : It is well known that the decomposition of $\mathrm{ZnO}$ substrates leads to an increase in $\mathrm{Zn}$ and $\mathrm{O}$ diffusion during high temperature growth which can result in poor epitaxial growth and degrade the film quality $[3,6,7]$. The intentionally doped Zn into GaN shifts the band-gap energy from blue to red emission, and this has been observed by numerous researchers [8-10]. Zn doping of InGaN shows values 0.4 0.5eV lower than the calculated and experimental band-gap energy of $\operatorname{In}_{\mathrm{x}} \mathbf{G a}_{1-\mathrm{x}} \mathrm{N}$ without $\mathrm{Zn}$ doping. Diffusion into the epilayer from the substrate has been demonstrated by secondary ion mass spectrometry (SIMS) depth profile of the GaN/ZnO interface $[11,12]$. As a result, the $P L$ related to $\mathrm{Zn}-/ \mathrm{O}$ impurities appears as a broad band, similar to the case with $\mathrm{Mg}$ and $\mathrm{Cd}$ which was due to a strong phonon coupling in the re-combinations involving a broad range of phonon energies [13-15].

Solution - Single GaN buffer with InGaN epilayer: Only the LT-GaN

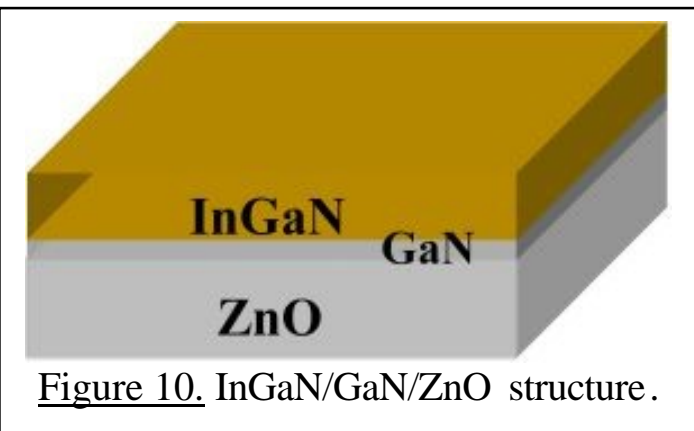

buffer layer was used and the high annealing temperature at $900^{\circ} \mathrm{C}$ was removed to avoid severe diffusion of $\mathrm{Zn}$ and $\mathrm{O}$, Figure 10. GaN has a smaller lattice mismatch with $\mathrm{ZnO}$ and lower bonding energy compared with AIN. Therefore, the crystal quality of LT-GaN as a buffer was expected to be better than that of LT-AIN. In addition, HT-GaN was eventually replaced with InGaN due to its lower growth temperature. These two approaches make it possible for crystalline InGaN layers as seen by XRD measurements. The optical microscope image reveals the transparency and color homogeneity of the top surface of the InGaN layer, as seen in Figure. 12.

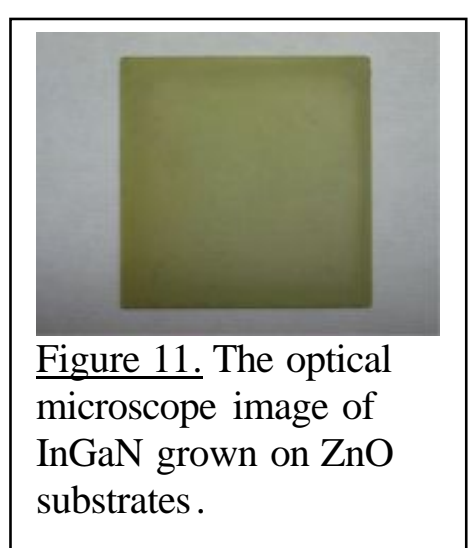

Three different generations of epitaxy were developed in this project. Generation 1 utilized a GaN nucleation layer followed by a $1000^{\circ} \mathrm{C}$ anneal before a $\mathrm{GaN}$ epilayer. Generation 1 also went further and added a second GaN buffer before the $1000^{\circ} \mathrm{C}$ anneal. The cracking issue for the growths was solved by the end of Generation 1. Generation 2 replaced the second GaN buffer with AIN and thereby solving the pinhole problems leading to no peeling off of the epi-layer. Generation 3 went back to just the one GaN buffer layer and InGaN was grown successfully on top.

Results from this project for both GaN and InGaN are compared with state-of-the-art epitaxy and other projects $[1,3,16,17]$. In this project, due to a limited range of growth temperatures; the crystal quality, optical performance, and surface roughness need to be 
further improved in order to be similar to standard GaN grown on sapphire, Table 2. When using MBE growth, the FWHM of (0002) plane of GaN on $\mathrm{ZnO}$ is higher than that of GaN grown on $\mathrm{ZnO}$ by MOCVD. However, the PL data shows a difference between MBE and MOCVD, but this is easy to understand. The broader PL peak of GaN on $\mathrm{ZnO}$ by MOCVD was due to the $\mathrm{Zn} / \mathrm{O}$ diffusion from $\mathrm{ZnO}$, which was grown at a higher temperature of $800^{\circ} \mathrm{C}$ compared to MBE.

The results for thick InGaN films in this project are comparable to standard InGaN layers grown on sapphire by MOCVD and are better than those reported for MBE growth, Table 3. The XRD FWHM value of InGaN on $\mathrm{ZnO}$ by MOCVD is much smaller than that by MBE. The roughness of the InGaN surface in this project is in the same range as those grown on sapphire and is smoother than those grown by MBE. However, PL results in this project are around 500aresec which is still slightly larger than that grown on sapphire by MOCVD due to $\mathrm{Zn} / \mathrm{O}$ incorpo ration into the epilayers. Here, it is interesting to point out that the phase separation in InGaN that is observed for high indium concentration on sapphire is not for $\mathrm{ZnO}$. Details are explained elsewhere in the report.

Table 2. MOCVD GaN results compared with state of the art and other projects.

\begin{tabular}{|l|c|c|c|}
\multicolumn{1}{|c}{ GaN } & $\begin{array}{c}\text { XRD (0002) } \\
\text { FWHM (arcsec) }\end{array}$ & FWHM (meV) & $\begin{array}{c}\text { Roughness } \\
(\mathbf{n m})\end{array}$ \\
\hline Sapphire & $200 \sim 300$ & $30 \sim 40$ & $0.5 \sim 1.0$ \\
\hline MOCVD & $\sim 452$ & $\sim 200$ & 20 \\
\hline MBE & $1200 \sim 1400[3]$ & $10 \sim 15[3]$ & $10 \sim 30[13]$ \\
\hline
\end{tabular}

Table 3. MOCVD InGaN results compared with state of the art and other projects.

\begin{tabular}{|l|c|c|c|}
\hline \multirow{2}{*}{ InGaN (>20\% In) } & XRD (0002) & \multicolumn{1}{c|}{ PL } & \multicolumn{1}{c|}{ Roughness } \\
\cline { 2 - 4 } FWHM (arcsec) & FWHM (meV) & $(\mathbf{n m})$ \\
\hline Sapphire & $\mathbf{4 0 0 ~ 5 0 0 [ 1 4 ]}$ & $\mathbf{3 5 0} \sim 400$ & $\mathbf{1 . 4 ~ 2 . 9}[14]$ \\
\hline MOCVD & $\sim 864$ & $\sim 500$ & $3.0 \sim 4.0$ \\
\hline MBE & $\mathbf{1 4 0 0 ~ 1 6 0 0 [ 1 2 ]}$ & no ref. & $5.7[3]$ \\
\hline
\end{tabular}

\section{Molecular Beam Epitaxy}

MBE allows for $\mathrm{H}_{2}$ to be used during GaN growth. In addition, growths were able to be done at a low temperature of $550^{\circ} \mathrm{C}$ compared to MOCVD temperature of greater than $1000^{\circ} \mathrm{C}$. However, even with these attributes, the samples grown were not uniform and had pits. Samples showed broad spectrums for both $\mathrm{GaN}$ and $\mathrm{InGaN}$ on $\mathrm{ZnO}$ as can be seen in Figure 12. 


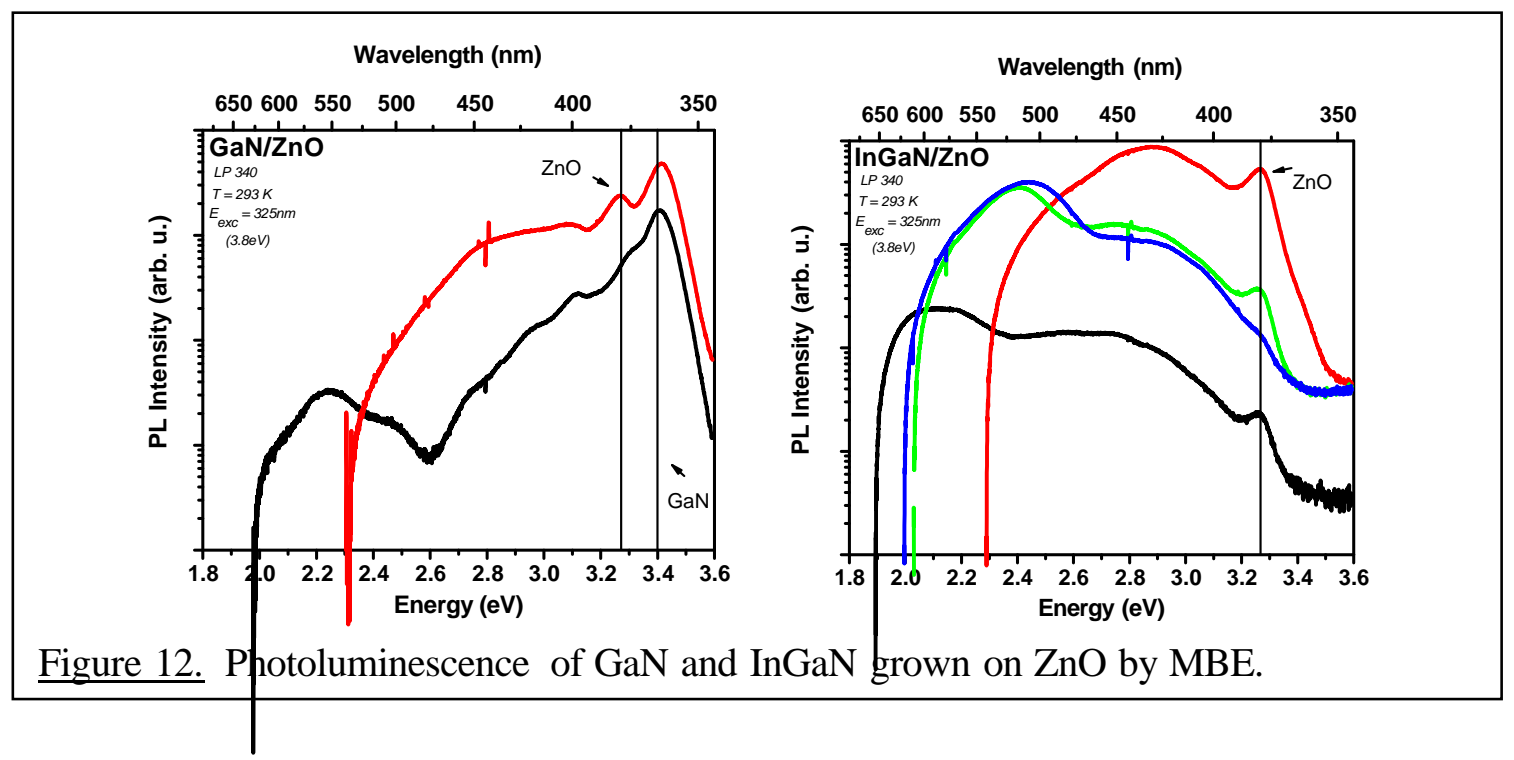

Growth Results on ZnO by MOCVD

Issues for MOCVD Growth

While there have been some reports of the growth of $\mathrm{GaN}$ based materials on $\mathrm{ZnO}$ by $\mathrm{MBE}$, there have been few using MOCVD. Since MOCVD is the dominant growth technology for GaN-based materials and devices, there is a need to more fully explore this technique for $\mathrm{ZnO}$ substrates. However, there are a number of obstacles that need to be addressed for the MOCVD growth

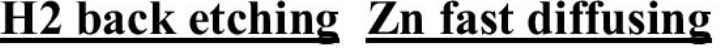

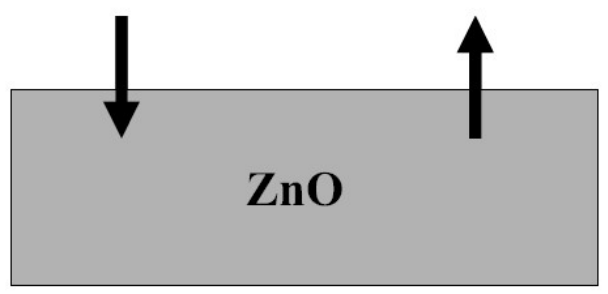

Figure 13. $\mathbf{H}_{2}$ back etching into substrate and $\mathrm{Zn}$ diffusing into epilayers causing poor epilayer growth of GaN. of $\mathrm{GaN}$ on $\mathrm{ZnO}$. These include the thermal stability of the $\mathrm{ZnO}$ substrate, the out-diffusion of $\mathrm{Zn}$ from the $\mathrm{ZnO}$ into the GaN, and $\mathrm{H}_{2}$ back etching of the substrate, Figure 13.

Commencial MOCVD usually grow over $1000^{\circ} \mathrm{C}$ for GaN on sapphire substrates, which makes it difficult for MOCVD growth of GaN and InGaN on $\mathrm{ZnO}$ substrates. So far, to our knowledge, only one group has reported the MOCVD growth of GaN on $\mathrm{ZnO}$, which showed XRD ( $2^{\text {nd }}$ order) and PL peaks from GaN layer [18], and there is no report found for MOCVD growth of InGaN on $\mathrm{ZnO}$. Poor surface preparation and severe issues associated with high temperature growth by MOCVD can degrade the GaN film quality. ZnO displays a much lower hardness compared to other materials at high temperature allowing it to decompose more easily than other available materials with a rise in temperature. The decomposition leads to an increase in $\mathrm{Zn}$ diffusion causing poor epilayer growth of GaN. 
An additional problem associated with high temperature growth is $\mathrm{H}_{2}$ back etching of $\mathrm{ZnO}$. An increase in temperature increase the $\mathbf{H}_{2}$ etching rate and damages the substrate before the epilayers can be deposited, Figure 14. $\mathrm{H}_{2}$ free growth has been investigated, but $\mathrm{Zn}$ diffusion has been found to occur through the epilayer under some growth conditions [19]. $\mathrm{NH}_{3}$ itself contributes $\mathrm{H}_{2}$ to the growth. At low temperatures around ${ }^{500}{ }^{\circ} \mathrm{C}$ the contribution is small and therefore not detrimental. As the temperature is ramped up to $600^{\circ} \mathrm{C}$ and higher, $\mathrm{NH}_{3}$ cracking efficiency increases quickly causing excess $\mathrm{H}_{2}$ to

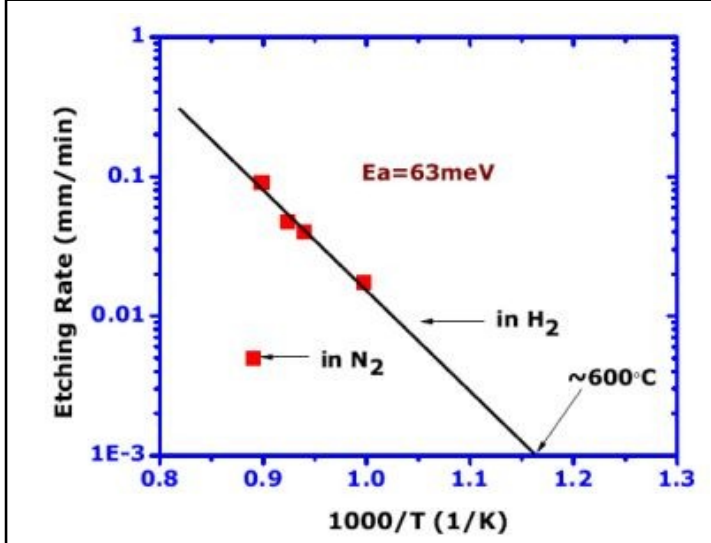

Figure 14. Anhenius plot for the $\mathrm{H}_{2}$ etching rate $(\mathrm{mm} / \mathrm{min})$ against the temperature $(1 / \mathrm{T})$.

etch the $\mathrm{ZnO}$ substrate. DMHy and TBHy have been investigated as $\mathrm{H}_{2}$-free $\mathrm{N}$ sources with low-temperature decomposition $\left(100 \%\right.$ below $\left.800^{\circ} \mathrm{C}\right)$, but both were found to cause high levels of carbon contamination making high quality GaN growth impossible [19].

Another issue is heterovalency at the $\mathrm{GaN} / \mathrm{ZnO}$ interface to enhance the

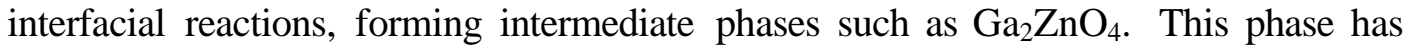
been identified with poor crystalline quality and diminishes the advantage of the lattice-matched $\mathrm{ZnO}$ substrate [2]. Theref ore, several studies used low temperature to solve this problem by pulse laser deposition (PLD) and plasma-assisted MBE.

Numerous studies have also reported that high-quality GaN films could be successfully grown on thermally treated $\mathrm{Zn}$ - and $\mathrm{O}$-face $\mathrm{ZnO}$ substrates and are able to avoid the intermediate phase by using MBE [3, 7]. However, T. Suzuki et al. found that the $\mathrm{Ga}_{2} \mathrm{O}_{3}$ and $\mathrm{Zn}_{3} \mathrm{~N}_{2}$ phases still appeared at the $\mathrm{GaN} / \mathrm{ZnO}$ interface by SIMS measurement, confirming significant interdiffusion [12]. From PLD results, the authors revealed terrace structures on the GaN surface and no interfacial layer existence under low temperature $[20,21]$. Nevertheless, this intermediate layer still occurred above $540^{\circ} \mathrm{C}$. As a result, the PLD and MBE techniques for growth of GaN on $\mathrm{ZnO}$ substrate are still limited by temperature control and growth rate.

\section{Generation 1a: High \\ Temperature Anneal of Low Temperature -GaN on $\mathrm{ZnO}$}

The goal of Generation 1a was to be able to grow $\mathrm{GaN}$ on $\mathrm{ZnO}$ at high temperature to achieve high quality GaN.

In order to grow high-quality

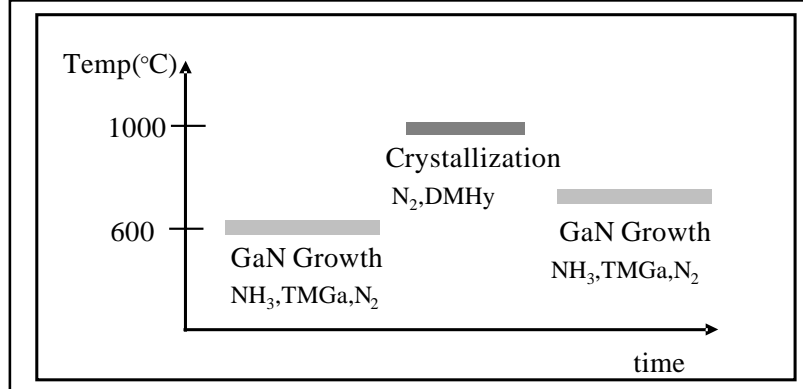

Figure 15. Schematic of growth condition for Generation 1a. 
GaN on $\mathrm{ZnO}$, growth must be done in a $\mathrm{N}_{2}$ atmosphere. However, $\mathrm{NH}_{3}$ cracking introduces unwanted $\mathrm{H}_{2}$ into the growth process at the desired high temperature. In order to overcome this DMHy was used as a replacement for the $\mathrm{N}_{2}$ source in this generation. Three steps are introduced to reach this high temperature while maintaining a stable substrate. The first step covered the $\mathrm{ZnO}$ surface with a thick low temperature (LT) layer. Then, recrystallization was done at a high temperature of $1000^{\circ} \mathrm{C}$. DMHy was used here as a protective gas preventing GaN from decomposition. GaN was then regrown after the annealing, see Figure 15.

Results showed serious surface cracking, Figure 16(a). However, after annealing optimization, PL and XRD did show GaN peaks even with surface cracking. Possible origins for the cracking are phase transformation and recrystallization during temperature ramping leading to a concentration of stress in the grain boundaries. $\mathrm{NH}_{3}$ can diffuse through the cracks into voids undemeath the cracks, degrading the film quality and making the high temperature epitaxy impossible. Calculations were done showing that the lattice mismatch and themal expansion differences are not the reason for the cracks, Figure 16(b).

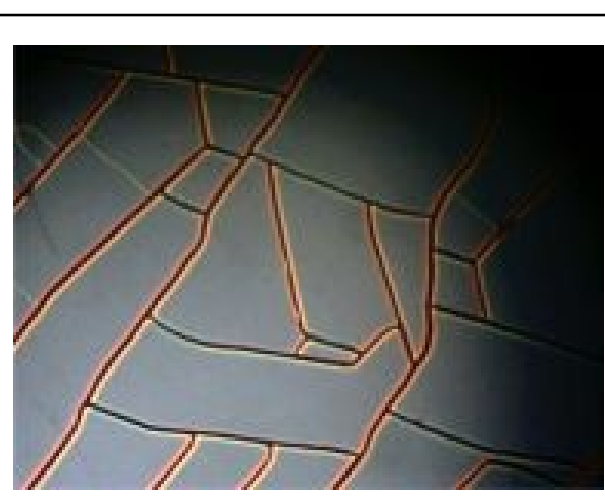

(a)

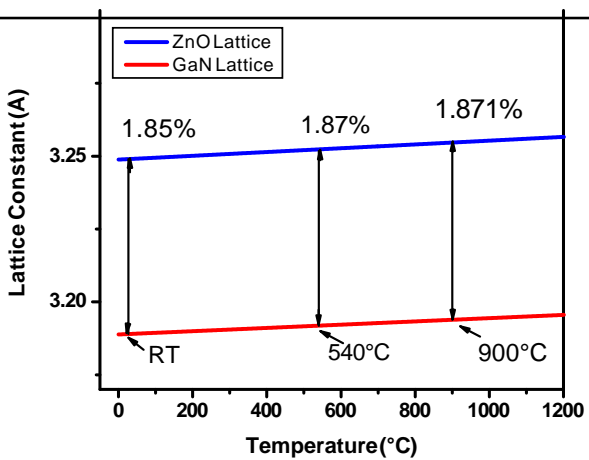

(b)

Figure 16. (a) Cracking on surface (b) lattice constant calculations between GaN and $\mathrm{ZnO}$.

Generation 1b: Multi LT-GaN Buffer for Crack Free Growth

The goal of Generation 1b was to solve the cracking problem in Generation 1a.

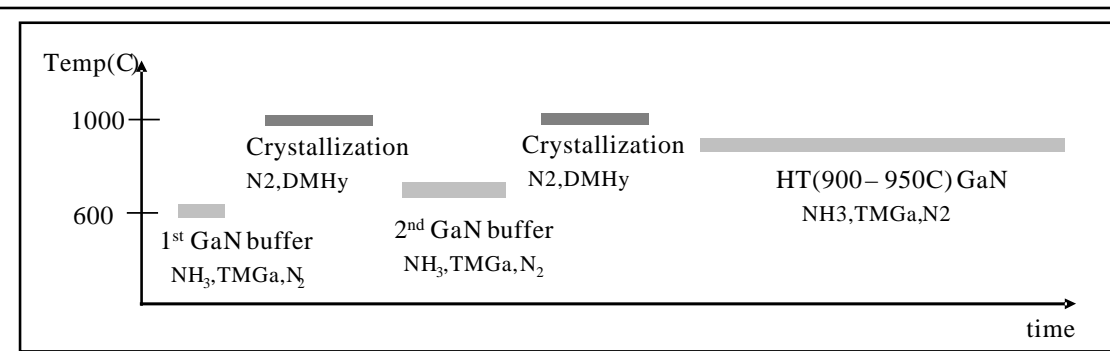

Figure 17. Schematic of growth condition for Generation $1 \mathrm{~b}$.

Generation 1b modified the first buffer layer from Generation 1a by making it thinner for easier phase transformation during the crystallization step and also for less 
stress between $\mathrm{ZnO}$ and the first buffer. Crystallization with DMHy is still done at $1000^{\circ} \mathrm{C}$ with the $2^{\text {nd }} \mathrm{GaN}$ buffer also the same as before. The $2^{\text {nd }} \mathrm{GaN}$ buffer layer covers the pinholes from the 1st GaN buffer and provides a second layer to ensure the surface is completely and uniformly covered by GaN before high-temperature growth, see Figure 17.

Results showed that with multiple GaN layers, the temperature was able to reach higher temperatures of $900-950^{\circ} \mathrm{C}$ which is useful for LED structures. The reflective

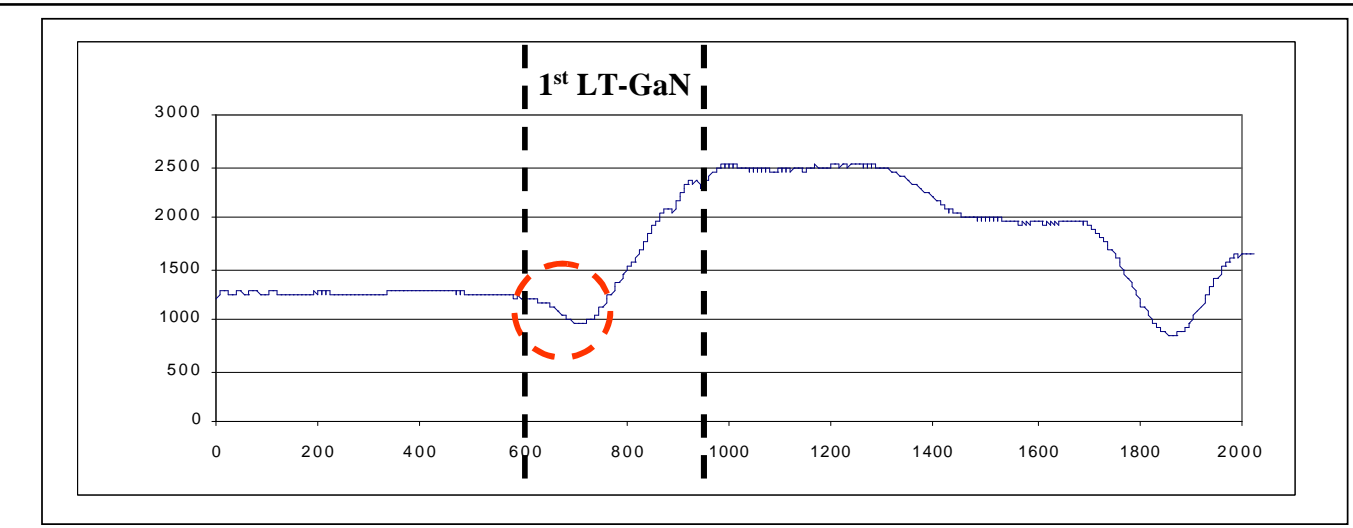

(a)

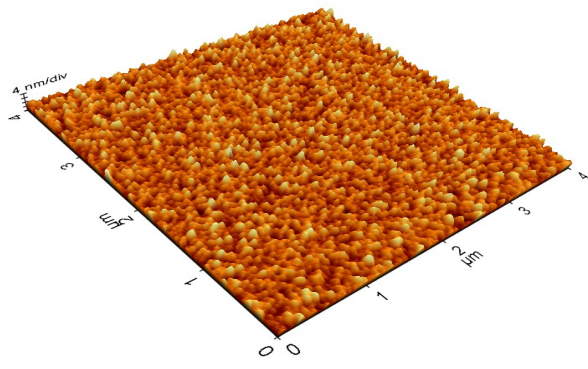

GaZnON layer

RMS = 1.5nm

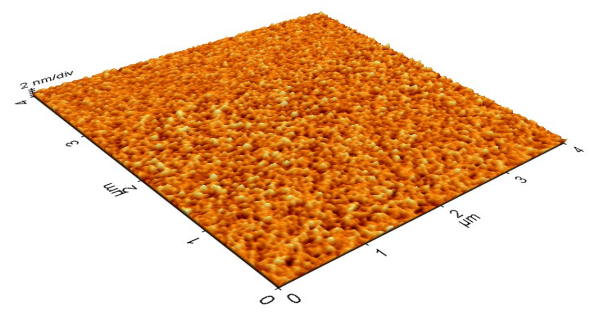

GaN layer

RMS = 1nm

(b)

Figure 18. (a) Epimetrix showing reflective signal going down at the initial stage of 1st LT-GaN. (b) AFM showing rougher surface for intermixed layer.

signal goes down at the initial stage of the $1^{\text {st }}$ LT-GaN layer, Figure 18(a). The signal drop is not due to surface roughing and therefore means the growth of a layer whose refractive index is smaller than $\mathrm{ZnO}$. There is a possibility of an intemixing layer between the $\mathrm{ZnO}$ and $\mathrm{GaN}$ at the initial stage, a GaZnNO quatemary layer. The signal drop was found to be very sensitive to the growth temperature of the $1^{\text {st }}$ GaN buffer layer. AFM data of a rougher surface for the intermixing layer when the signal drops is shown in Figure 18(b).

After optimization, a sweet spot was found for the transition layer for crack free growth. The transition layer is sensitive to growth temperature and helps with 
preventing cracks by absorbing the strain between the $\mathrm{ZnO}$ and $\mathrm{GaN}$. However, some regions showed peeling-off which could be attributed to a thick intermixing GaZnNO layer, causing an adhesion problem. All areas also showed high density etching pits of $\mathrm{ZnO}$ through GaN pinholes.

A possible origin of the peeling off and etching pits are the pinholes in the LTGaN multi-buffer layers. $\mathrm{NH}_{3}$ can attack the $\mathrm{ZnO}$ substrate through the pinholes. At the end of Generation 1b, the cracking issues were solved, but pinholes still appeared in the samples. Therefore, high density pinhole regions are the peeling off regions and in order to solve the peeling off issue, the pinhole density in the LT multi buffer layer needed to be reduced. Images of peeling off of the $\mathrm{ZnO}$ substrate with SEM images of the pinholes in the sample are shown in Figure 19.
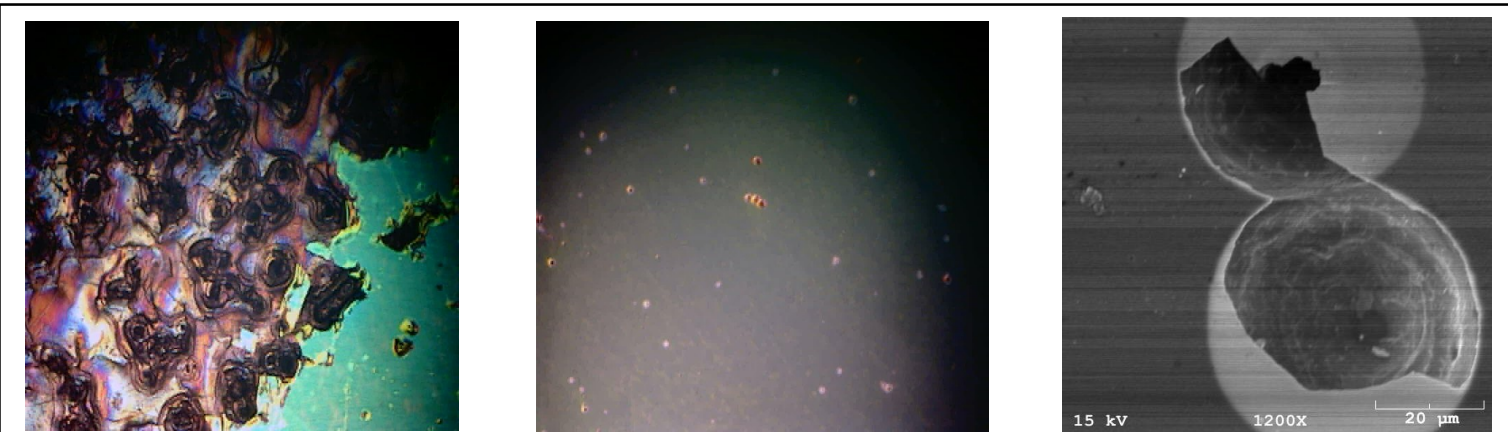

Figure 19. (a) Peeling-off region, (b) point etching of $\mathrm{ZnO}$, and (c) SEM of point etching of $\mathrm{ZnO}$.

Generation 2: $1^{\text {st }}$ AlN \& $2^{\text {nd }}$ GaN LT-buffer

The goal of Generation $\mathbf{2}$ is to solve the peeling off issue from Generation $\mathbf{1 b}$.

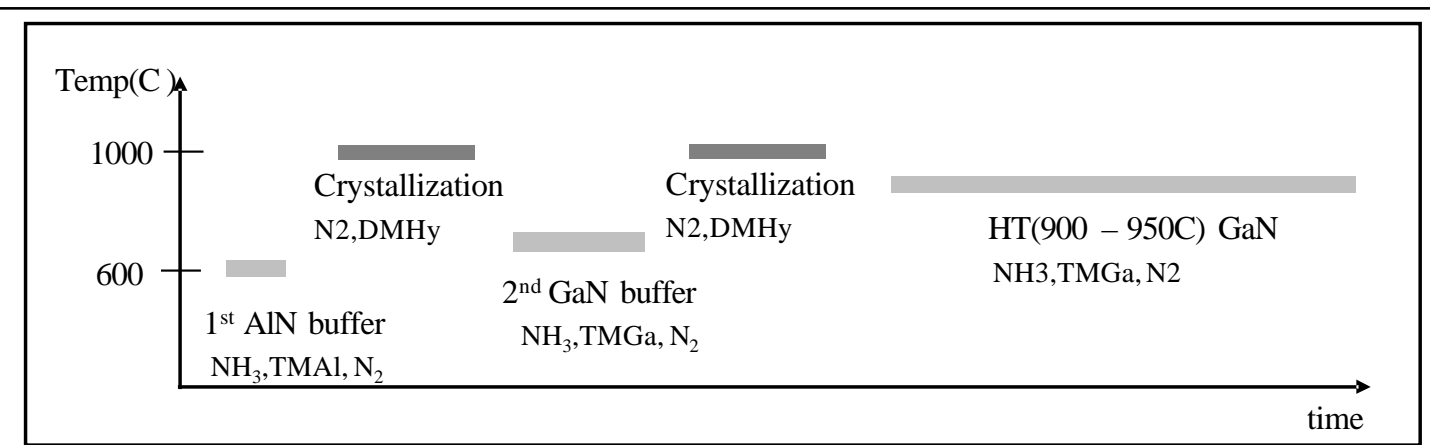

Figure 20. Schematic of growth condition for Generation 2.

In Generation 2 only the first buffer layer was changed as was in Generation $1 \mathbf{b}$, Figure 20. Instead of using GaN, AIN was used to help with preventing peeling off due to Al's low surface mobility and high surface coverage. AIN provides a more thorough surface coverage on $\mathrm{ZnO}$ allowing for lower surface pinholes during the 
high temperature crystallization step. It has a stronger bond than Ga forming a higher stable interfacial layer for high temperature growth.

Results showed PL and XRD with FWHM of 151 arcsec, Figure 21. An optimal point was found for no cracking or peeling-off. The AIN buffer provided a more densely covered nuclei on top of the $\mathrm{ZnO}$ surface and the interfacial layer of $\mathrm{AlZnNO}$ probably is more suitable for absorbing the strain than GaZnNO.

By the end of Generation 2, the EPD for GaN on $\mathrm{ZnO}$ was found to be $1.2 \times 10^{6} \mathrm{~cm}^{-2}$. Therefore, lower defect GaN on $\mathrm{ZnO}$ can be achieved compared to $\mathrm{Al}_{2} \mathrm{O}_{3}$ and SiC. Blue emission was also observed from the samples on different

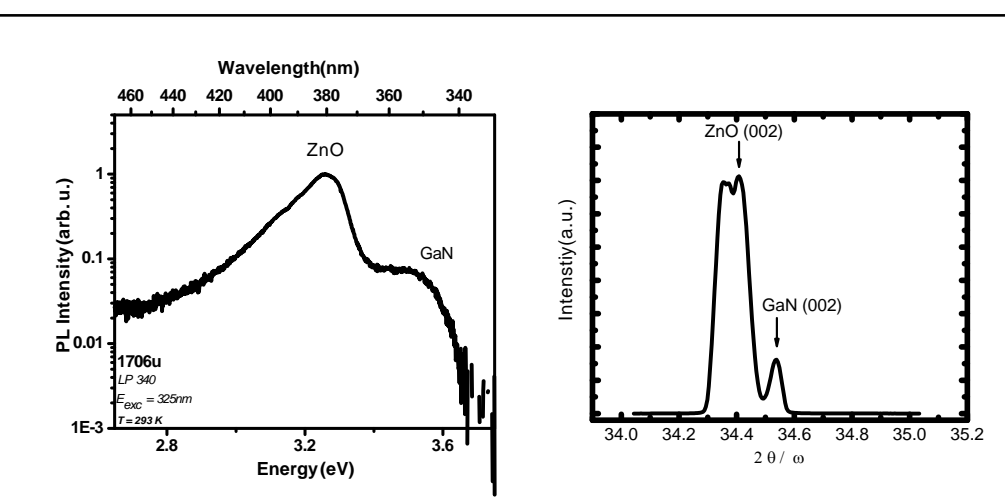

Figure 21. PL and XRD of GaN on $\mathrm{ZnO}$. areas of 20x20 $\mathrm{mm}^{2}$ samples, Figure 22. Crack free, no peeling-off, and low defect density GaN surface have been obtained, however, XRD and PL still do not show good quality results.

\section{Generation 3: LT-GaN Buffer and InGaN Layers}

The goal of Generation 3 is to optimize the characteristics of the grown layers for both GaN and InGaN.

In this section, the successful growth of InGaN layers on (0001) $\mathrm{ZnO}$ substrates by MOCVD is reported. The grown InGaN layers contained high In composition in the range of 17$27 \%$, which are suitable for typical InGaN multiple quantum well LED device applications. It is demonstrated that the key factor of this

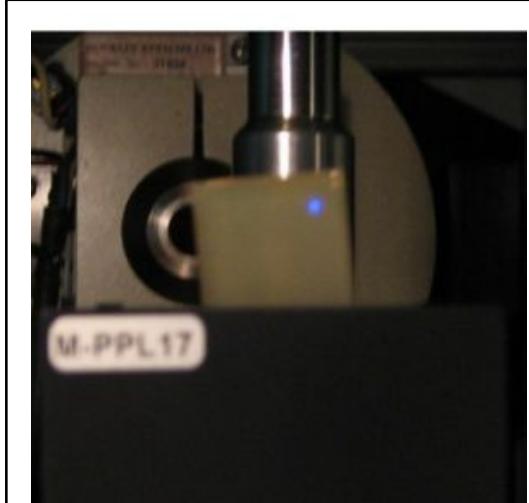

Figure 22. Blue emission from GaN on $\mathrm{ZnO}$. success is the use of a low temperature GaN buffer layer to grow InGaN films on ZnO substrates.

\section{Experimental Procedures for InGaN growth}

InGaN films were grown on the $\mathrm{Zn}$ face of (0001) $\mathrm{ZnO}$ substrates by MOCVD in a modified commercial rotating disk reactor with dual injector blocks. A low temperature GaN buffer layer was grown at $530^{\circ} \mathrm{C}$ with a thickness of about $30 \mathrm{~nm}$ using trimethylgallium (TMGa) and ammonia $\left(\mathrm{NH}_{3}\right)$ as the gallium and nitrogen sources, respectively. Following the buffer, InGaN layers of about $70 \mathrm{~nm}$ thick were 
grown at temperature s ranging from $680^{\circ} \mathrm{C}$ to $720^{\circ} \mathrm{C}$ by introducing trimethylindium (TMIn) and triethylgallium (TEGa) into the reactor. $\mathrm{N}_{2}$ carnier gas was used during the whole growth process in order to avoid etching of the $\mathrm{ZnO}$ surface. By varying the growth temperature, the In concentration and growth rate of the investigated films were varied from $17 \%$ to $27 \%$ and 0.16 to $0.2 \mu \mathrm{m} / \mathrm{h}$, respectively.

\section{Thin LT-GaN buffer and thick InGaN layer achieved around $700^{\circ} \mathrm{C}$}

Generation 3 used a GaN buffer instead of an AIN buffer and had no additional buffer layers like other generations. GaN has a lower bonding energy than AIN leading to a better quality buffer layer. In addition, the lattice mismatch between $\mathrm{ZnO}$ and GaN is only $1.9 \%$. The InGaN films were grown at a shorter time and lower temperature than GaN allowing for less $\mathrm{H}_{2}$ etching from $\mathrm{NH}_{3}$ on the $\mathrm{ZnO}$ surface. Good quality InGaN films were able to be achieved due to the shorter time and lower temperature growth. The annealing process was also removed to avoid carbon contamination from DMHy. The growth schematic and the in-situ reflectance curve of the typical growth procedure are shown in Figure 23(a) and (b).

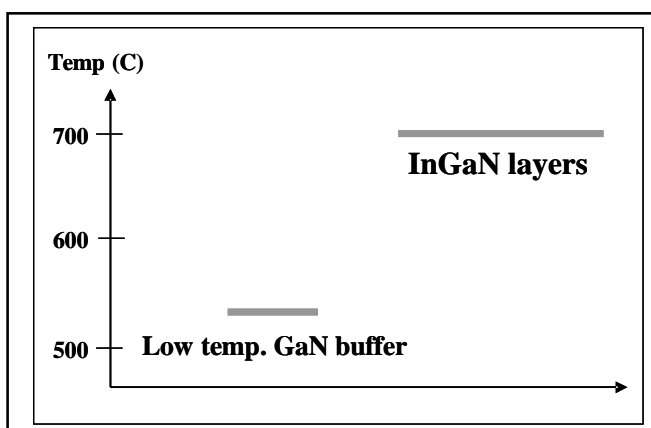

(a)

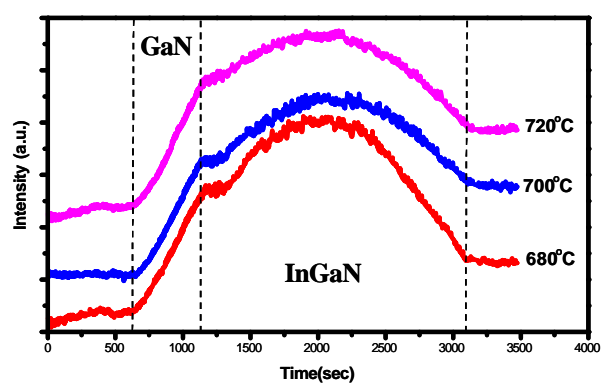

(b)

Figure 23. (a) Illustration of the growth procedure (b) In-situ reflectance curve of the typical growth procedure.

It clearly demonstrates the growth of LT-GaN buffer and the growth of InGaN sequentially. The InGaN gnowth rate increased as temperature decreased at a speed of about $0.16 \sim 0.2 \mathrm{um} / \mathrm{h}$ in a temperature range of $680 \sim 720^{\circ} \mathrm{C}$.

\section{Crack free, no peeling-off, and low defect density InGaN surface}

The optical microscope image of the InGaN surface is shown in Figure 24(a). The image shows a smooth and mirror-like surface. No cracking or peeling-off can be seen compared with previous generations. This can be attributed to shorter time and lower growth temperature, but also because the InGaN layer is more ductile than the GaN layer. The inserted image also shows the whole $20 \times 20 \mathrm{~mm}^{2} \mathrm{InGaN}$ film grown on $\mathrm{ZnO}$ wafer. SEM shows the surface microstructures of the GaN buffer and the InGaN film, Figure 24(b) and (c), respectively. The GaN buffer reveals the quadrilateral grains and the InGaN layer shows link-strip grains. 


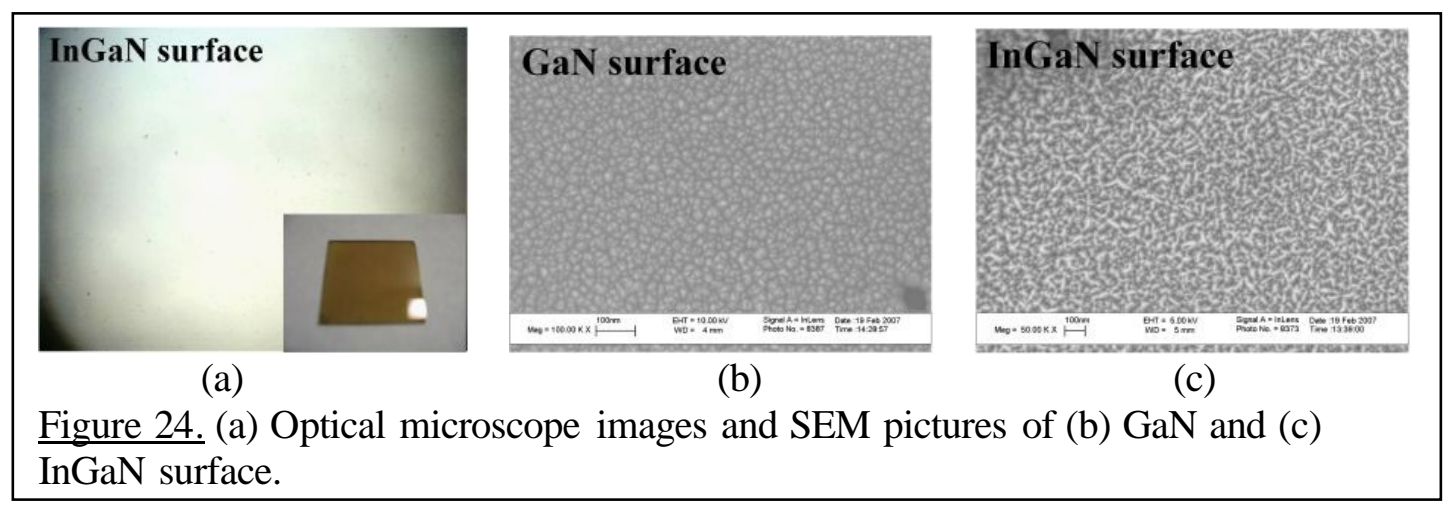

Structure and In composition of InGaN layers characterized by HRXRD

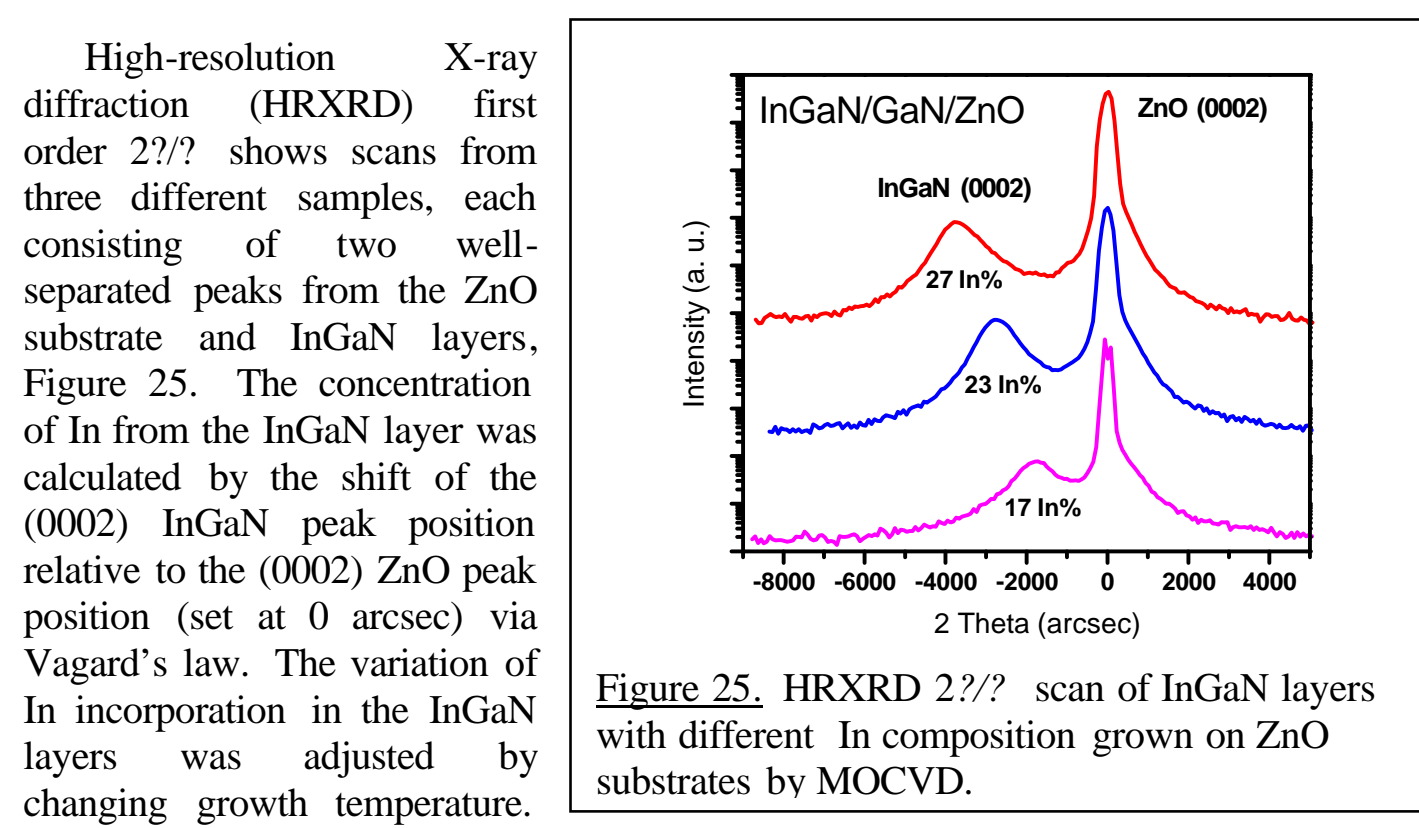

The InGaN films showed single crystal diffraction peaks by 2?/? scans corresponding to the $17 \%, 23 \%$, and $27 \%$ In-content with the samples grown at $720^{\circ} \mathrm{C}, 700^{\circ} \mathrm{C}$, and $680^{\circ} \mathrm{C}$, respectively. All XRD patterns revealed the small shoulder close neighbor on the right of the $\mathrm{ZnO}$ peaks which might be from diffraction of the GaN buffer layer. Remarkably, only InGaN and $\mathrm{ZnO}$ peaks appeared in all the samples and no extra peaks were observed even in samples with composition as high as $27 \%$. This meant no In droplets or phase separation were detected by XRD from the InGaN layers. In addition, all samples have been examined by FE-SEM with mirror-like InGaN surfaces and no evidence of In droplets on the surface.

For InGaN grown on GaN template coated sapphire, the phase separation was widely reported for high In composition, caused by spinodal decomposition. However, for our InGaN films grown on $\mathrm{ZnO}$ substrate, In droplets or phase separation were not detected by HRXRD, in contrast to the above cases [22-25]. From theoretical calculation and experimental observation, biaxial strain in the epilayer is helpful in suppressing the phase separation in InGaN materials [26, 27]. 
Phase separation easily occurs when the InGaN layer starts to relax due to compressive strain from GaN/sapphire. Here, the observed suppression of phase separation in the film is believed to be due to a higher strain state of InGaN with high In content compared with the InGaN/GaN template/sapphire materials system. The InGaN layer may stay completely strained on the thin GaN buffer, which is coherently grown on the underlying $\mathrm{ZnO}$, with high In composition since InGaN consisting of $18 \% \mathrm{In}$ is exactly lattice-matched with $\mathrm{ZnO}$. As a result, a higher strain state in InGaN films with high In concentration will be provided by $\mathrm{ZnO}$ compared with those grown on GaN/sapphire to retard the phase separation. This discrepancy is possibly caused from the different strain relaxation mechanisms in thin GaN/ZnO and GaN template/sapphire substrate.

\section{Optical characteristic of InGaN by $P L$}

Room temperature

photoluminescence (PL) spectra of the same three samples as xray are show in Figure 26. The emissions from the InGaN layers with different In compositions and from the $\mathrm{ZnO}$ substrate are observed. For the samples with In composition of $17 \%, 23 \%$, and $27 \%$, the emission peaks were observed at $2.4,2.1$, and $1.9 \mathrm{eV}$, respectively. Assuming the $70 \mathrm{~nm}$ InGaN layers were strained and employing the reported band gap expression, $E g=3.42-(0.65) x$ $3.4159 x(1-x)$ [28], where $x$ is the composition of In, the band edge emission energy can be calculated if the In concentration is known. Using the $x(I n)$ values of $17 \%, 23 \%$, and $27 \%$ from HRXRD measurements in Figure

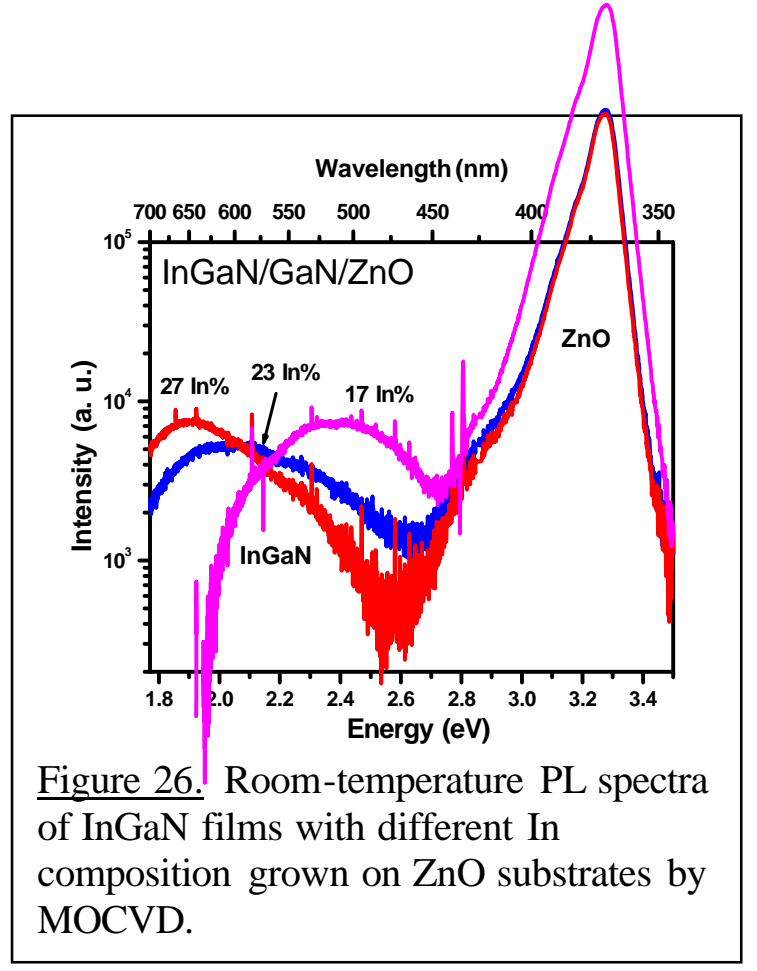
26, the calculated PL peak energies of $2.8,2.6$, and $2.5 \mathrm{eV}$ were obtained, respectively, which are quite different from the expenimental PL results in Figure 27. Each calculated PL peak for the InGaN layer shifts from the experimental spectral PL peak by about 0.4 0.6eV. The lower energy PL emissions may be due to $\mathrm{Zn}$ and $\mathrm{O}$ diffusion into the InGaN layers forming impurity levels inside the band gap emitting light with energy lower than the InGaN energy gap. A high density diffusion of $\mathrm{Zn}$ and $O$ impurities into InGaN might also be a contributing factor to the broad full width half maximum seen in PL. 
To further verify the InGaN related PL emission, temperature-dependant $P L$ was done over a wide range of temperature $s$ from $80 \mathrm{~K}$ to $673 \mathrm{~K}$ for the InGaN film grown at $700^{\circ} \mathrm{C}$, with $\mathrm{x}(\mathrm{In})$ of $23 \%$, Figure 27. The variation in intensity of InGaN (below 630 nm) with temperature is seen in this region. It shows that the PL intensity decreases with an increase in temperature, slowly at the low temperature region of $80-300 \mathrm{~K}$ but rapidly at the high temperature region of 373673K. Figure 28 exhibits the

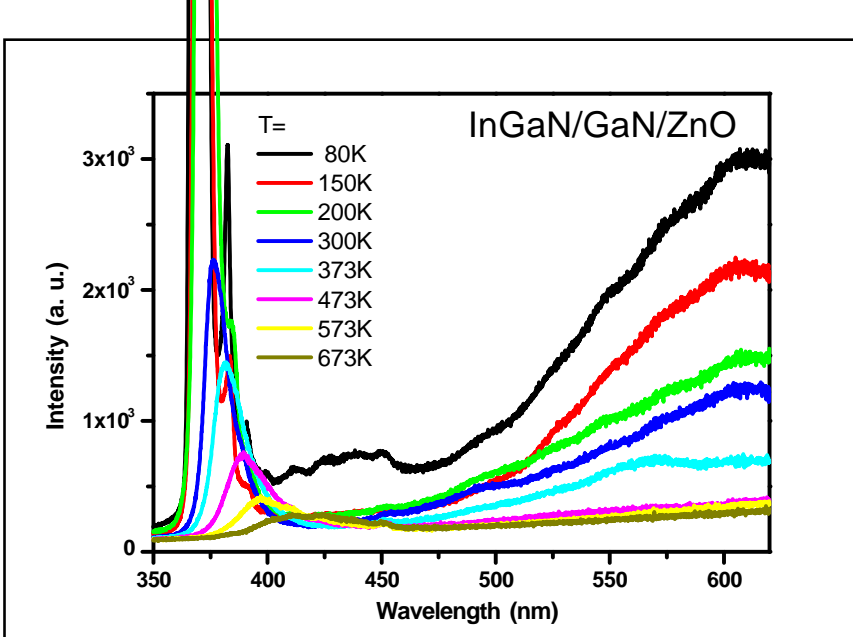

Figure 27. Temperature -dependent PL spectra (80-673 K) of InGaN film grown on $\mathrm{ZnO}$ substrate at $700{ }^{\circ} \mathrm{C}$.

Anhenius plot of InGaN-related PL peak intensity versus temperature in the range of 80-673K. Best fitting leads to an activation energy, $E_{a}$, of about $59 \mathrm{meV}$ for InGaN with $23 \%$ In content. Smith et al. [29] reports an $E_{a}=56 \mathrm{meV}$ for $\operatorname{In}_{\mathrm{x}} \mathrm{Ga}_{1-\mathrm{x}} \mathrm{N}$ epilayer with $x=0.12$ and Teo et al. [30] reports on $I_{0.2} G_{a}{ }_{0.8} N$ MQWs with an $E_{a}$ value of 63 $\mathrm{meV}$. The data reported in this study is close to the above reported values in literature.

\section{Evolution of surface}

morphology with temperature

The surface roughness can be improved by increasing growth temp. Increased temperature changes the growth mode from grain to step-like as seen in the AFM images shown in Figure 29. At high temperature s, the InGaN layer is grown in a stepflow like mode, which is a duplication of the underlying GaN buffer. At a low temperature, the InGaN surface is rough, which means growth of InGaN is featured by the nucleation of discrete islands and coalescence of the islands subsequently. This growth mode results in poor quality films because dislocations and other crystalline imperfection will form at the grain boundaries. Therefore, in onder to obtain high quality InGaN films, temperature must be controlled higher than $720^{\circ} \mathrm{C}$. The AFM results show the surface roughness was around $1-2 \mathrm{~nm}$ at high growth temperature.

\section{Phase Separation}

InGaN grown on sapphire have shown phase separation as reported in other literature. However, no phase separation was seen in the same growth on $\mathrm{ZnO}$ substrates.

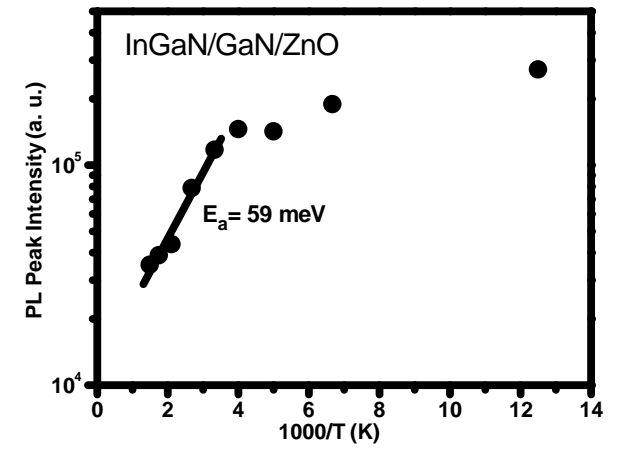

Figure 28. PL intensity versus temperature (80-673 K) of the InGaN layer. 


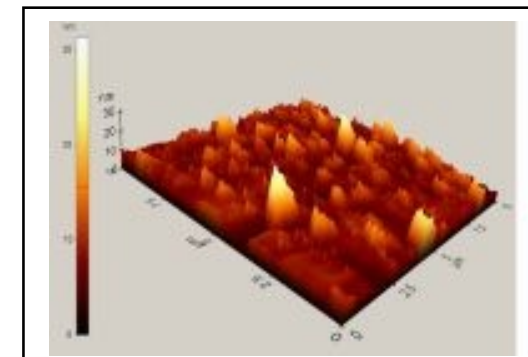

(a)

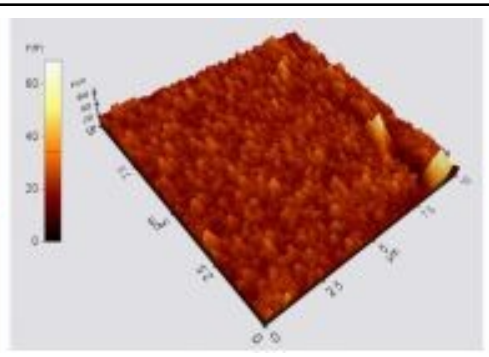

(b)

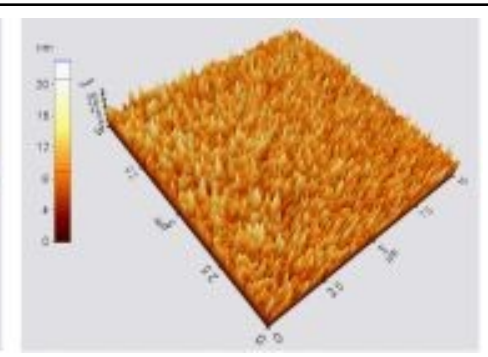

(c)

Figure 29. AMF images $(10 \mu \mathrm{m} \times 10 \mu \mathrm{m})$ for $\mathrm{InGaN}$ sample grown at (a) $680{ }^{\circ} \mathrm{C}$, b) 700 ${ }^{\circ} \mathrm{C}$, and c) $720{ }^{\circ} \mathrm{C}$.

The optimization of InGaN alloys is important for the development of optical devices operating in the green light. However, the epitaxial growth of InGaN has always been a challenge at high In concentration. The large atomic size difference between Ga and In atoms leads themodynamics to predict a miscibility gap between $\mathrm{GaN}$ and $\mathrm{InN}$ [6]. Low luminescence efficiency has been observed for green light emitting InGaN quntum wells (QWs) with submicron-sized small phase separation [7]. For InGaN grown on GaN template coated sapphire, phase separation is frequently observed in the bulk layers as well as in the QWs, especially for higher In composition (>20\%), [8-12] caused by spinodal decomposition.

In our previous study, successful growth of InGaN layers on (0001) $\mathrm{ZnO}$ substrates by MOCVD have been reported. For InGaN grown on sapphire, phase separation will happen when In compositions exceed certain levels in the alloys leading to nonhomogeneity [13]. This study addresses the issues of InGaN phase separation and introduces the $\mathrm{ZnO}$ substrate as a growth medium for obtaining In concentration up to $42 \%$ without any detectable phase separation.

\section{Experimental Procedures}

For

preparation of the GaN templates, the 2 um thick GaN was grown on (0001)

sapphire

substrates

$1020^{\circ} \mathrm{C}$ using $\mathrm{H}_{2}$ carrier gas, trimethyl gallium (TMGa) and

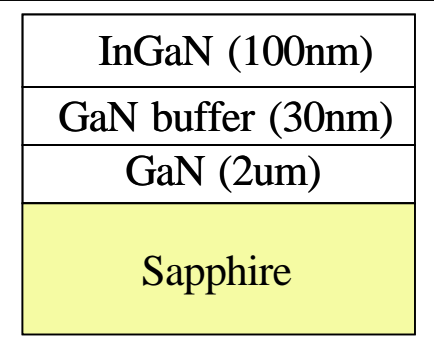

(a)

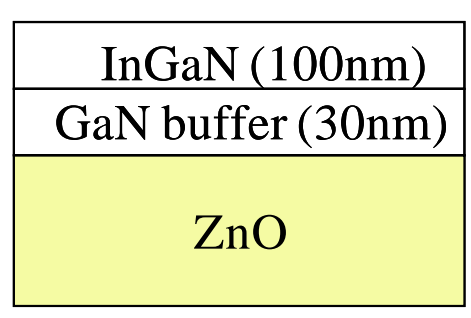

(b)
Figure 30. Schematic illustration of the two sample structures: (a) InGaN/GaN/GaN template/sapphire and (b) InGaN/GaN/ZnO.

ammonia $\left(\mathrm{NH}_{3}\right)$ as the gallium and nitrogen sources, respectively. The InGaN/GaN structures were grown on the (0002) GaN/sapphire and $\mathrm{Zn}$ face of (0001) $\mathrm{ZnO}$ substrates using the same growth conditions by MOCVD in a modified commencial rotating disk reactor with dual injector blocks. Schematic illustrations of the grown structure for sapphire and $\mathrm{ZnO}$ substrates are shown in Figure 30. A low temperature GaN buffer 
layer was grown at $5^{\circ} 0^{\circ} \mathrm{C}$ with a thickness of about $30 \mathrm{~nm}$ using $\mathrm{TMGa}$ and $\mathrm{NH}_{3}$ as the gallium and nitrogen sources, respectively. Following the buffer, InGaN films of about $100 \mathrm{~nm}$ thick were grown at temperatures ranging from $660^{\circ} \mathrm{C}$ to $740^{\circ} \mathrm{C}$ by introducing trimethylindium (TMIn) and triethylgallium (TEGa) into the reactor. $\mathrm{N}_{2}$ carrier gas was used during the whole growth process in order to avoid etching of the $\mathrm{ZnO}$ surface.

\section{Suppression of Phase Separation in InGaN Grown on Lattice-Matched ZnO Substrates}

The HRXRD ( $1^{\text {st }}$ onder) 2?/? scan pattems from the InGaN layers on sapphire and $\mathrm{ZnO}$ substrates are shown in Figure 31 (a) and (b), respectively. The indium composition in the layers was calculated by the shift of the (0002) InGaN peak position relative to the (0002) GaN and (0002) $\mathrm{ZnO}$ peak positions (set at 0 arcsec) via Vagard's law. Figure 31 (a) shows a single InGaN phase corresponding to $17 \%$ In-content for samples grown at

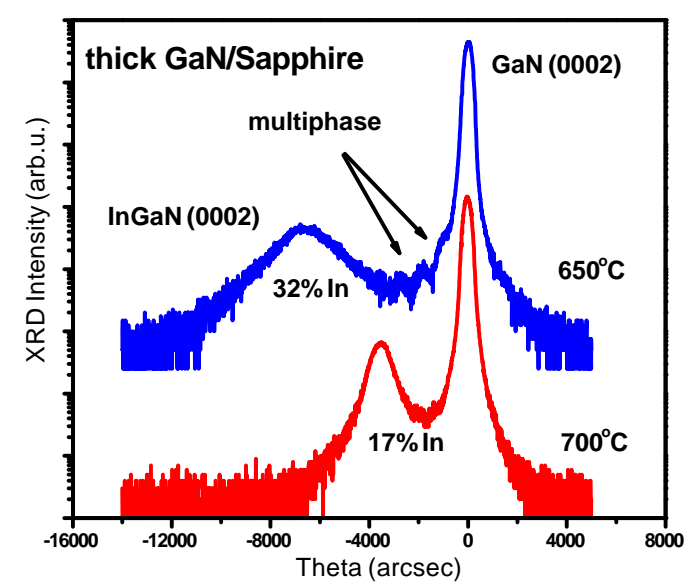

(a)

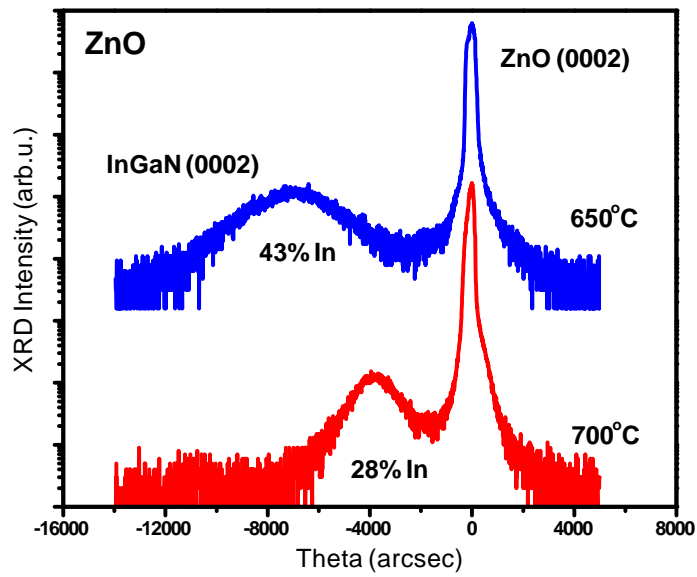

(b)

Figure 31. HRXRD 2?/? scan of InGaN layens with different In composition grown on (a) thick GaN/sapphire and (b) ZnO substrates by MOCVD.

emperature of $700^{\circ} \mathrm{C}$. However, when the growth temperature was ramped down to $650^{\circ} \mathrm{C}$ multiple phases of InGaN appeared with the highest concentration at $32 \% \mathrm{In}$. From themodynamics, an increase in In concentration resulting in phase separation is attributed to the growth of InGaN alloys below the critical temperature required for miscibility. This growth condition falls into the region of spinodal decomposition in the GaN-InN phase diagram for relaxed layers [23, 26]. The rapid increase in lattice mismatch between InGaN and thick GaN/sapphire will easily generate edge dislocations to relax the compressive strain when In composition increases. As a result, the compressive strain relaxation is progressive and phase separation occurred at growth temperatures as low as $650^{\circ} \mathrm{C}$. Remarkably, only single InGaN phases appeared on $\mathrm{ZnO}$ substrates with compositions as high as $28 \%$ and $43 \%$ In grown at temperatures of 700 C and $650^{\circ} \mathrm{C}$, respectively, Figure 31(b). It is found that InGaN with $43 \%$ In is located at a stable region for miscibility in the GaN-InN phase diagram in the strained layer case [26]. This location indicates that In incorporation can be as high as $60 \%$ around $650^{\circ} \mathrm{C}$ without spinodal decomposition [26]. From theoretical calculation and experimental observation, biaxial strain in the epilayer is helpful in suppressing the phase separation in InGaN 
materials [26, 27, 31]. Phase separation easily occurs when the InGaN layer starts to experience compressive strain relaxation from thick GaN/sapphire. It is believed that the InGaN layers grown on $\mathrm{ZnO}$ might stay at a higher strain state than that grown on sapphire.

It has been widely reported that the compressive strain can be reduced by $\mathrm{Si}$ doping into the GaN based epilayer [32-34]. I. H. Lee et al., reported that Si-doping induced relaxation of compressed strain in GaN layers [32]. M. K. Chen and co-workers proposed that spinodal decomposition is strongly caused by different Si doping as seen in changes in InGaN strain relaxation of InGaN/GaN MQWs [34]. P. Cantu and his group also found that increasing $\mathrm{Si}$ doping enhanced the relaxation of compressively strained $200 \mathrm{~nm}$-thick $\mathrm{Al}_{0.49} \mathrm{Ga}_{0.51} \mathrm{~N}$ layers grown on top of $1 \mu \mathrm{m}$-thick $\mathrm{Al}_{0.62} \mathrm{Ga}_{0.38} \mathrm{~N}$ coated sapphire [33]. Figure 32 shows the diffraction pattem of InGaN layers with Si doping grown at $700^{\circ} \mathrm{C}$ and $720^{\circ} \mathrm{C}$ on (a) sapphire and (b) $\mathrm{ZnO}$ substrates. Both InGaN films on sapphire and $\mathrm{ZnO}$ substrates show no phase separation under a growth temperature of

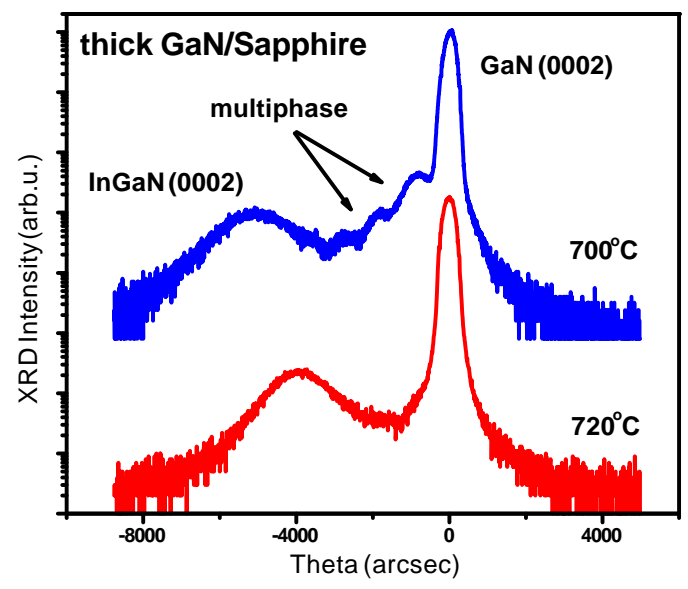

(a)

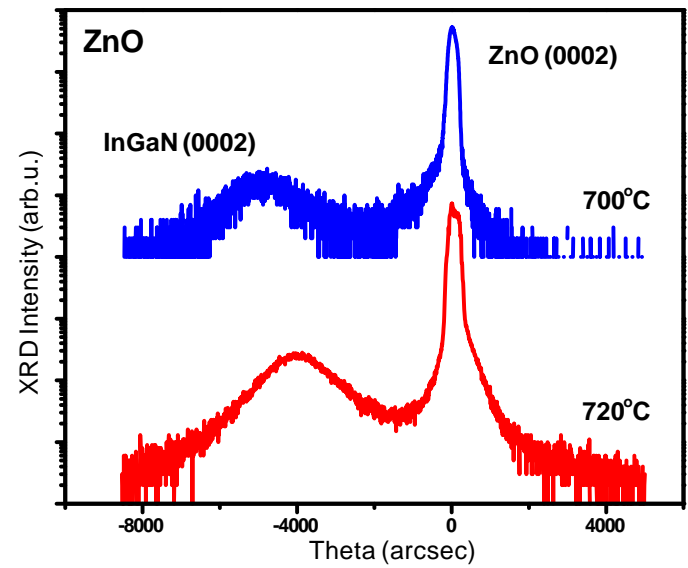

(b)

Figure 32. HRXRD 2?/? scan of InGaN layers with $\mathrm{Si}$ doping grown on (a) thick GaN/sapphire and (b) ZnO substrates by MOCVD.

$7^{\circ} \mathrm{C}$. However, as the growth temperature decreases to $700^{\circ} \mathrm{C}$, the diffraction pattem of InGaN layers on (a) sapphire shows multiple phases but on (b) $\mathrm{ZnO}$ shows no phase separation. The relaxation of the compressive strain is due to the substitution of larger Ga or In atoms by smaller Si atoms in the InGaN films. For the Si doped InGaN grown on sapphire at $720^{\circ} \mathrm{C}$, a certain strain level is preserved after Si doping due to an initial higher strain because of a smaller lattice mismatch between InGaN and thick GaN/sapphire at lower In concentration. Therefore, the phase separation cannot be observed. However, the initial strain level was reduced due to a larger lattice mismatch when the In content increased at a lower temperature of $700^{\circ} \mathrm{C}$. Therefore, compared to Figure 31 where no phase separation appeared at $700^{\circ} \mathrm{C}, \mathrm{Si}$ can further induce phase separation on sapphire. In contrast, the $\mathrm{Si}$ doped $\mathrm{InGaN}$ grown on $\mathrm{ZnO}$ at $700^{\circ} \mathrm{C}$ did not show multiple phases in HRXRD. This result indicates that $\mathrm{ZnO}$ can provide a higher strain state in InGaN films to suppress phase separation caused by Si doping compared to sapphire. 


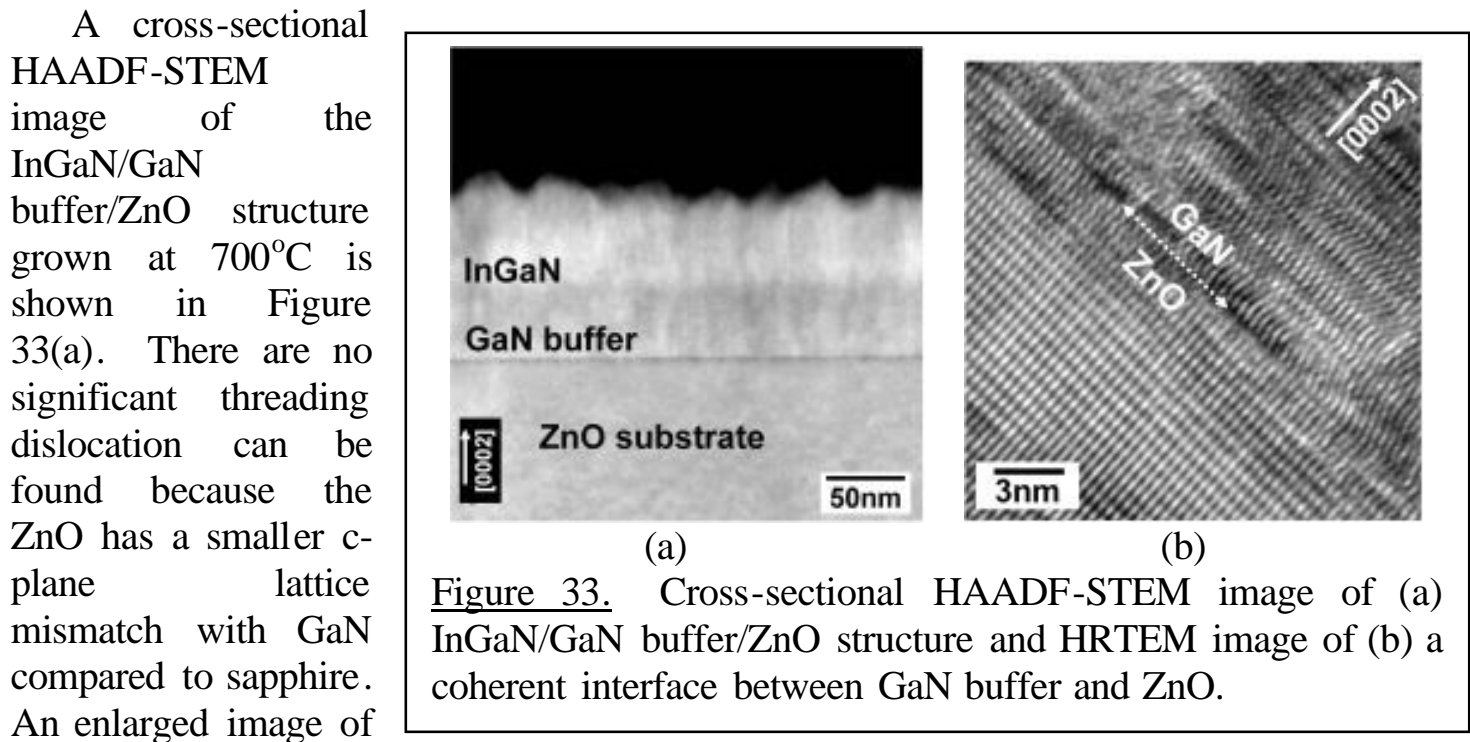

the lattices of the GaN buffer and $\mathrm{ZnO}$ substrate is shown in Figure 33(b). As can be seen, the two crystals match perfectly so that the lattice is continuous across the interface plane, which certainly shows that the interfaces between $\mathrm{GaN}$ and $\mathrm{ZnO}$ are coherent. A fully coherent interface with a slight mismatch of $1.8 \%$ leads to coherency strain in the adjoining lattices between the $\mathrm{GaN}$ buffer and $\mathrm{ZnO}$. In other words, the larger a-axis lattice constant of $\mathrm{GaN}$ on $\mathrm{ZnO}$ is preferable for lattice matching to $\mathrm{InGaN}$ with high $\mathrm{In}$ concentration. In addition, InGaN consisting of $22 \%$ In-content is exactly latticematched with $\mathrm{ZnO}$. As a result, the InGaN layer may stay completely strained on a thin GaN buffer, which is coherently grown on the underlying $\mathrm{ZnO}$, at high In composition since. In contrast, the large lattice mismatch between InGaN and thick GaN/sapphire will easily generate local dislocation, thereby, relaxing the compressive strain and inducing phase separation when In composition is at a high level. Therefore, a higher strain state in InGaN films with high In concentration $(28 \%, 43 \%)$ will be provided by $\mathrm{ZnO}$ compared with those $(17 \%$ 32\%) grown on thick GaN/sapphire.

The phase separation phenomena between both the $\mathrm{ZnO}$ and sapphire case can be addressed by previous studies on thick InGaN layers grown on GaN coated sapphire substrates also done in Dr. Ferguson's lab. A plot of a and c lattice constant versus In composition is shown in Figure 34. Data showed that In concentration

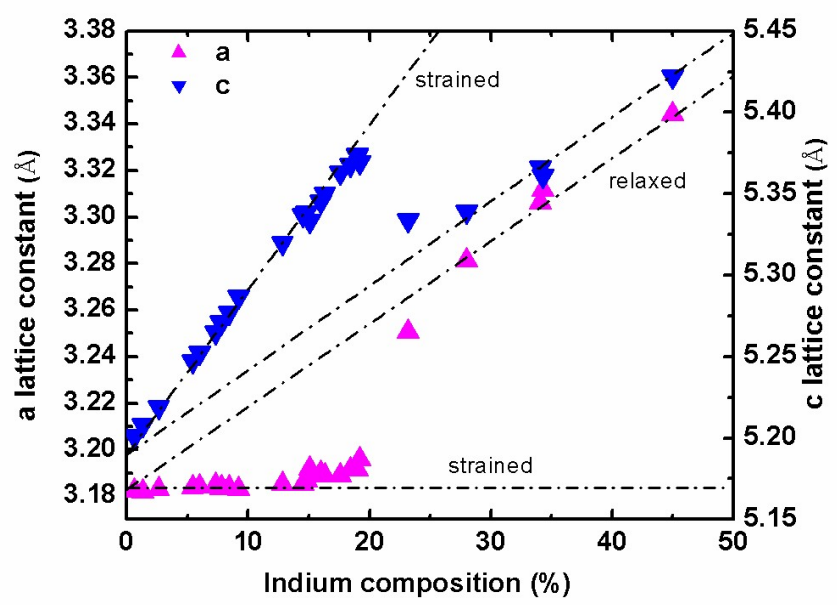

Figure 34. Plot of a and c lattice constant versus In composition. 
less than $20 \%$ in InGaN layers are coherently strained on the GaN templates. For In composition between $20 \%$ and $30 \%$, the layers start to gradually relax the strain, and for composition over $30 \%$ InGaN films appear to be fully relaxed. The different strain relaxation appears to be the reason that phase separation is observed for InGaN grown on sapphire compare d with $\mathrm{ZnO}$.

The lattice constant has been shown as a function of In composition, i.e. the strain state will be changed with In composition, which will be demonstrated by reciprocal space mapping (RSM), as shown in Figure 35. From it, two lines are drawn: the line to the origin, which is the zeno strain line where InGaN is fully relaxed, and the perpendicular line, where InGaN is fully strained on GaN. Clearly the InGaN layer with $x=0.14$ is coherently grown on

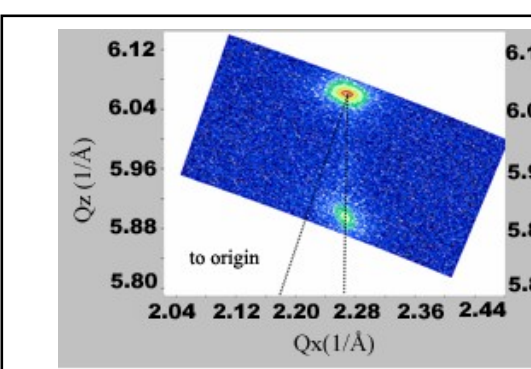

(a) $x=0.14$

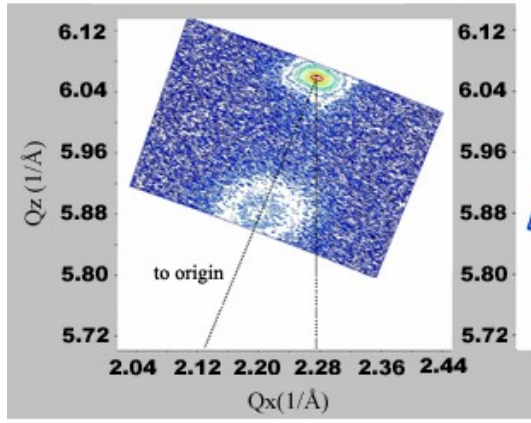

(c) $x=0.31$

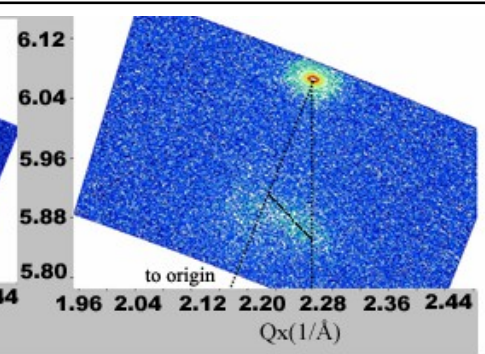

(b) $x=0.20$

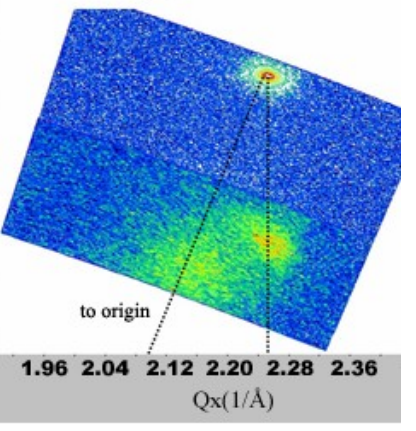

(d) $x_{1}=0.18 x_{2}=0.40$

Figure 35. Reciprocal space mapping of InGaN on sapphire.

the GaN template, whereas the InGaN layer with $x=0.31$ is fully relaxed. The InGaN layer with $20 \%$ indium is believed to undergo the strain relaxation process. A $20 \%$ isocomposition line connecting the fully strained to fully relaxed regions is drawn in Figure 35(b) for comparison. It is observed that the InGaN layer has different strain states continuously changing from fully strained to fully relaxed, while the composition remains almost unvarying. Therefore the strain relaxation is progressive and may take place either along the growth direction or across the hetero-interface.

From theoretical calculation and expenimental observation, biaxial strain in the epilayer is helpful in suppressing the phase separation in InGaN materials. Phase separation easily occurs when the InGaN layer starts to relax due to compressive strain from GaN/sapphire. For InGaN grown on $\mathrm{ZnO}$, there is a direct lattice match at $18 \%$ In which means that this coherent interface can incorporate more In composition than sapphire can. InGaN does not start to relax or become fully relaxed even at In percentages as high as $43 \%$. Phase separation issues can be significantly improved by growing on a $\mathrm{ZnO}$ substrate. Here, the observed suppression of phase separation in the film is believed to be due to a higher strain state of InGaN with high In content compared with the InGaN/GaN template/sapphire materials system. The InGaN layer may stay completely strained on a thin GaN buffer, which is coherently grown on the underlying 
$\mathrm{ZnO}$, with high In composition since InGaN consisting of $18 \%$ In is exactly lattice matched with ZnO. As a result, a higher strain state in InGaN films with high In concentration will be provided by $\mathrm{ZnO}$ compared with those grown on $\mathrm{GaN} /$ sapphire to retard the phase separation. This discrepancy is possibly caused from the different strain relaxation mechanisms in $\mathrm{ZnO}$ and $\mathrm{GaN}$ template/sapphi re substrate. In other words, the large lattice mismatch between InGaN and GaN template/sapphire will easily generate the local dislocation for relaxing the compressive strain to induce the phase separation when In compositi on is at a certain high level. A summary of In composition with strain limitations is illustrated in Figure 36.

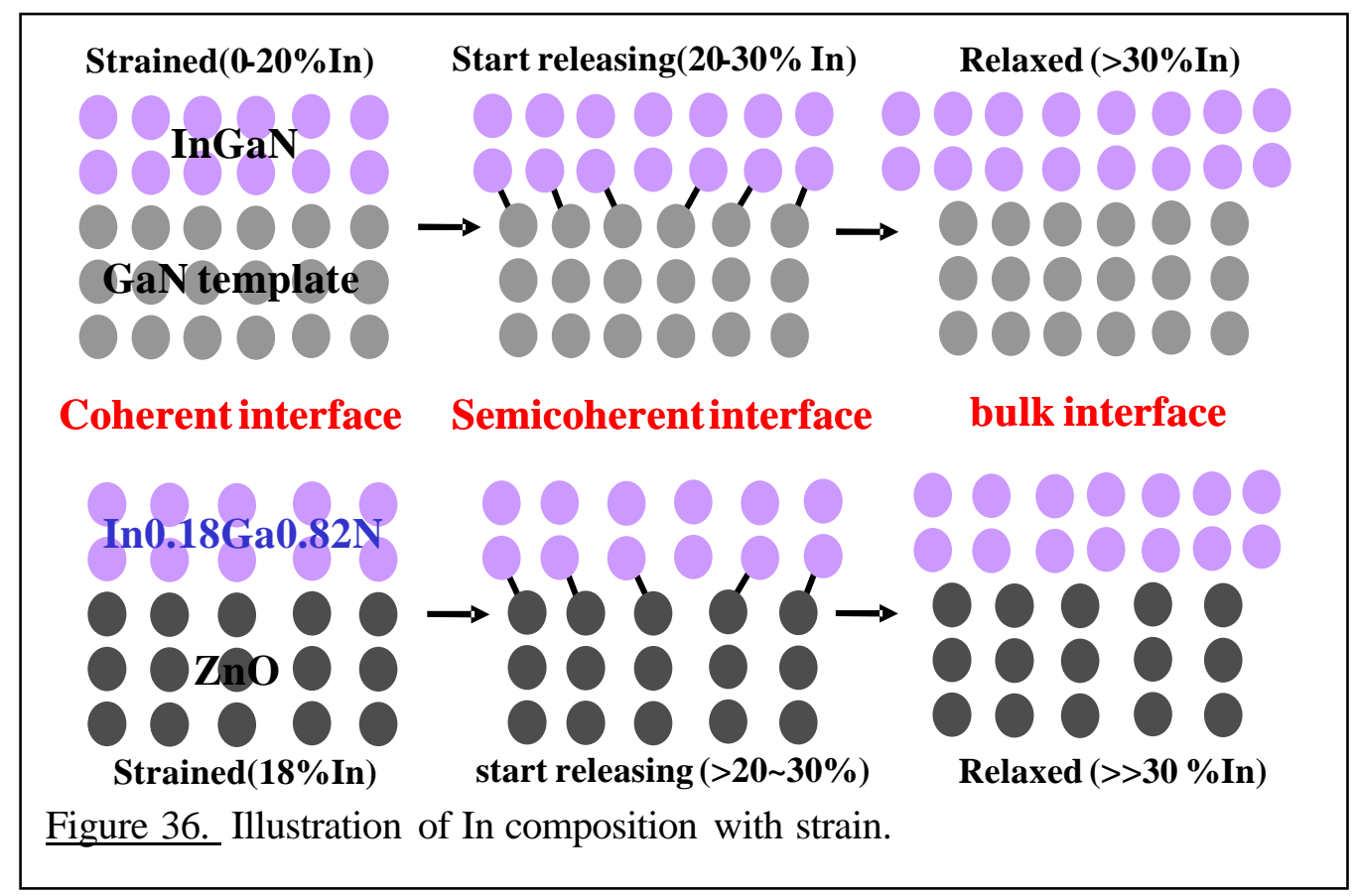

Direct Growths of GaN and InGaN on ZnO

GaN and InGaN were also directly grown on $\mathrm{ZnO}$. Figure 37 shows the schematics of the GaN/ZnO and InGaN/ZnO structures.

HRXRD reveals the single crystal diffraction GaN peak neighbors on the right of the $\mathrm{ZnO}$ peak, as shown in Figure 38. However, the roughness of the GaN surface still needs to be improved. Furthermore, the diffraction pattems of InGaN layers grown on $\mathrm{ZnO}$ substrates have been shown in Figure 39. The three samples all show multiple phases of InGaN with growth temperature from $680^{\circ} \mathrm{C}$ to $720^{\circ} \mathrm{C}$, from left to right. The multiple phases might be from a grown $\mathrm{InN}$ layer on $\mathrm{ZnO}$, which could 

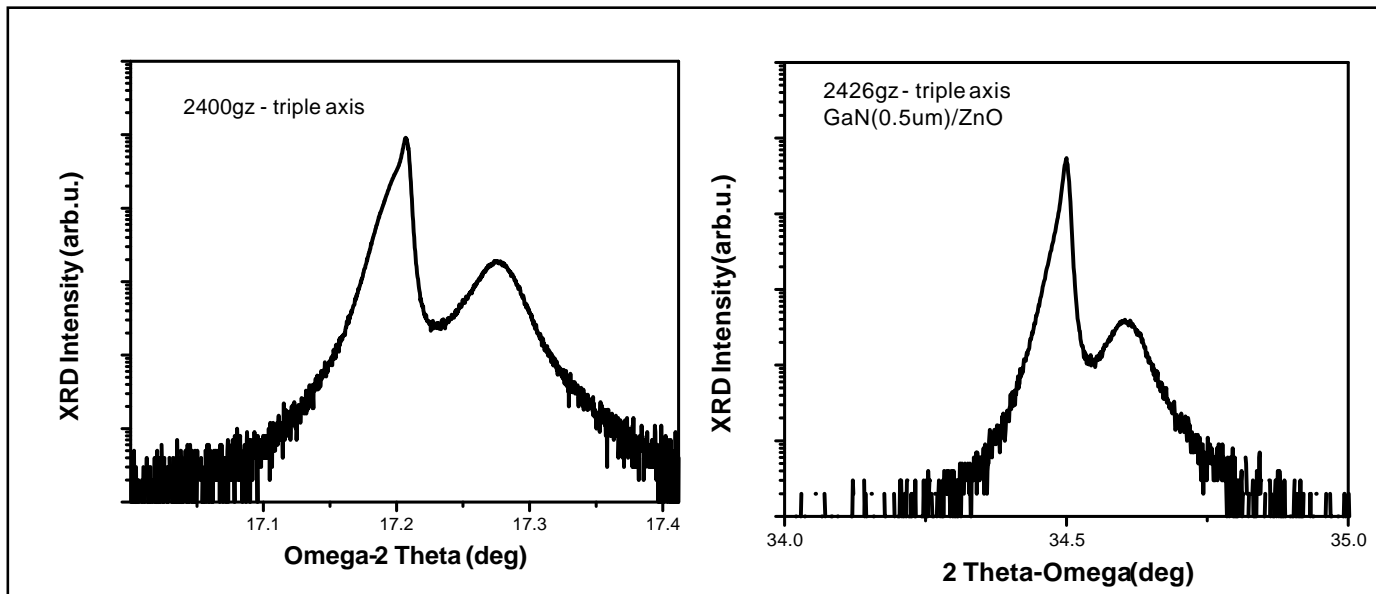

Figure 38. HRXRD of GaN directly grown on $\mathrm{ZnO}$.

provide the lower strain state for epilayers due to the large lattice mismatch between InN and InGaN. The large lattice mismatch between InGaN and InN can induce the phase separation. Therefore, further study is needed to understand how to directly grow InGaN without showing any phase separation.
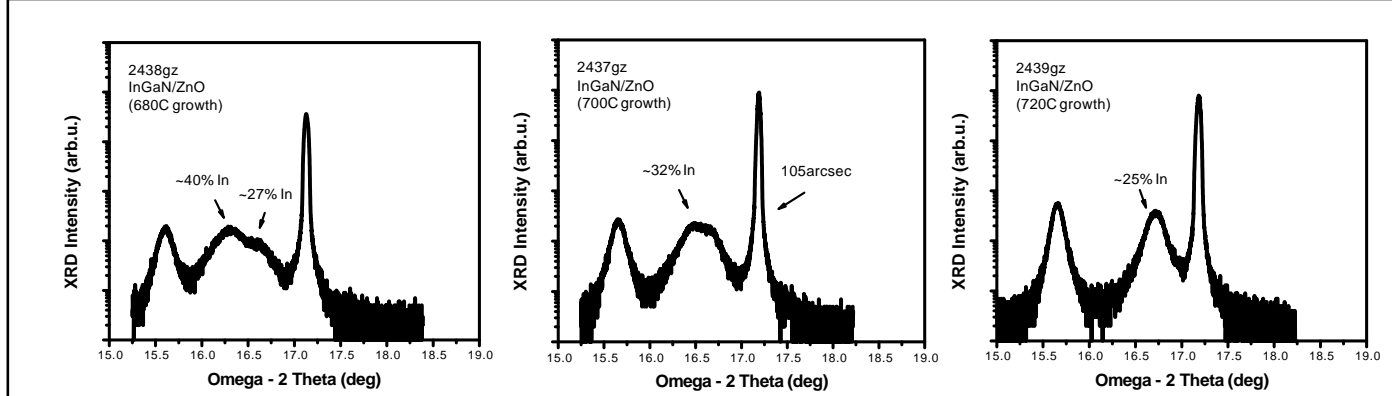

Figure 39. HRXRD of InGaN directly grown on $\mathrm{ZnO}$ showing multiple phases of InGaN.

Secondary Ion Mass Spectrometry (SIMS)

SIMS has been employed for concentration of different elements for growth in the $\mathrm{GaN} / \mathrm{ZnO}$ and $\mathrm{InGaN} / \mathrm{GaN} / \mathrm{ZnO}$ structures. A high concentration of $\mathrm{Zn}=5 \times 10^{19}$ and $\mathrm{O}=10^{21}$ atoms $/ \mathrm{cm}^{3}$ in the GaN layer indicates that there are a lot of $\mathrm{Zn}$ and $\mathrm{O}$ diffusion from the $\mathrm{ZnO}$ into the epilayers, Figure 40(a). The precise chemical stoichiometry of GaN grown on $\mathrm{ZnO}$ can be observed. The results also demonstrated that GaN has been successfully grown on $\mathrm{ZnO}$ substrates. High In content in InGaN was also verified by the SIMS concentration profile for the InGaN/GaN/ZnO structure, Figure 40(b). However, the severe diffusion of $\mathrm{Zn} / \mathrm{O}$ from the $\mathrm{ZnO}$ substrate $\left(\mathrm{Zn}=10^{20}, \mathrm{O}=10^{21}\right.$ atoms $\left./ \mathrm{cm}^{3}\right)$ still occurred, says that further improvement is needed to prevent the out-diffusion of $\mathrm{Zn}$ and $O$ from the $\mathrm{ZnO}$ substrate. In addition, the up-hill diffusion of $\mathrm{Zn}$ atoms was observed between the InGaN/GaN interface. 


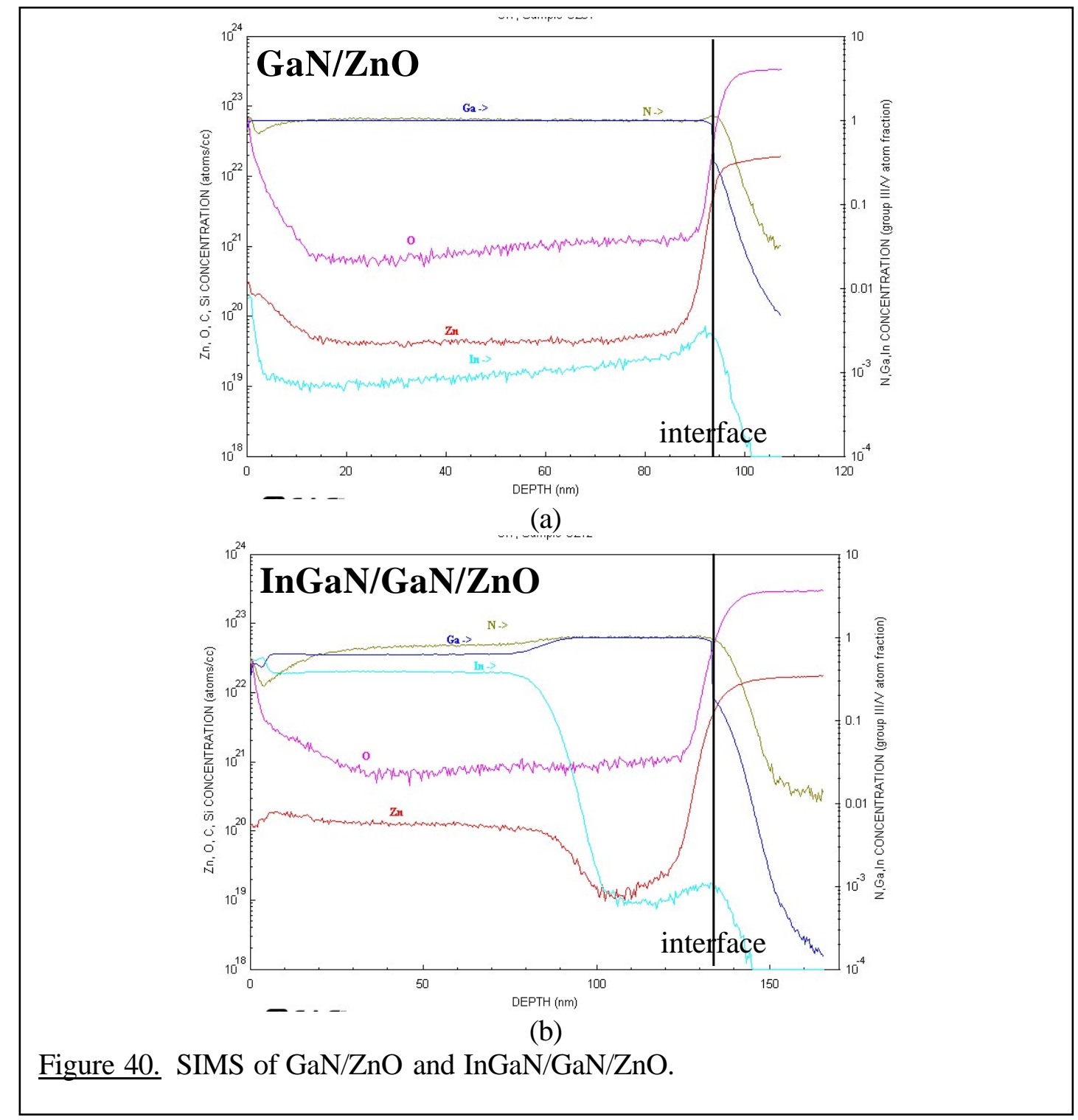

\section{Growth Results}

MOCVD technology has been employed for the epitaxial growth of InGaN layers on (0001) $\mathrm{ZnO}$ substrates. The key factor of this success is the use of a low temperature $\left(530^{\circ} \mathrm{C}\right)$ grown GaN buffer layer. Good quality $\mathrm{InGaN}$ films with a range of high $\mathrm{In}$ composition of $17-27 \%$, determined by high-resolution $\mathrm{X}$-ray diffraction, have been obtained with growth temperatures of $680-720^{\circ} \mathrm{C}$. The InGaN films show no In droplets or phase separation, which can be attributed to the strain relaxation in InGaN epilayers grown on $\mathrm{ZnO}$ substrates. Photoluminescence data for all InGaN films show a strong emission band from $\mathrm{ZnO}$ and broad InGaN-related emissions with the peak energy varying with In-composition. Peak energy values of the broad emissions are measured to be less than the calculated InGaN band gap by $0.5 \pm 0.1 e V$. These InGaN-related emissions may be due to recombinations involving $\mathrm{Zn}$-/O-impurities in InGaN due to the 
$\mathrm{Zn} / \mathrm{O}$ diffusion from the $\mathrm{ZnO}$ substrate. Temperature dependent PL measurements, 80$673 \mathrm{~K}$, obtained an activation energy of $59 \mathrm{meV}$ for the InGaN epilayer.

Beginning generations showed success in high temperature $\left(950^{\circ} \mathrm{C}\right)$ growth with $\mathrm{NH}_{3}$ for GaN growth on $\mathrm{ZnO}$ substrate by MOCVD. A detailed understanding of the interaction between GaN and $\mathrm{ZnO}$ during MOCVD growth was developed with a LTAIN/GaN multi-buffer method. Studies were done on the formation and role of the intermediate layer during the 1st LT-buffer growth. Solutions were found for the cracking and peeling-off issues. PL and XRD both showed peaks for GaN growth for these beginning generations. From these findings, a $\mathrm{ZnO}$ substrate technology has been developed for GaN optoelectron ics with low defect GaN on $\mathrm{ZnO}$ produced by MOCVD. Blue luminescence was also observed from the GaN film.

\section{Production of Phosphor Free White LED}

The device was based on a single InGaN well capped by the p-type GaN layer and the n-type $\mathrm{ZnO}$ layer (formed by the substrate, figure 41). The electrical properties of the active layers in the LED structure were characterized and compared with layers grown on sapphire. The p-type GaN on $\mathrm{ZnO}$ had a carier concentration of $5 \times 10^{17} \mathrm{~cm}^{-3}$ and mobility of $5 \mathrm{~cm}^{2} / \mathrm{V} . \mathrm{s}$, while the p-type GaN on sapphire had a carrier concentration of $2 \times 10^{18} \mathrm{~cm}^{-3}$ and mobility of $9 \mathrm{~cm}^{2} / \mathrm{V}$.s. Typical I-V characteristics are shown in figure 42, with a tum on voltage of just less than 3 volts. The resulting electroluminescence is shown in figure 43. Even at low cuments, the emission is visible under ambient conditions. The device is emitting a blend of blue light and a broad spectrum "yellow" band emission, creating white light.
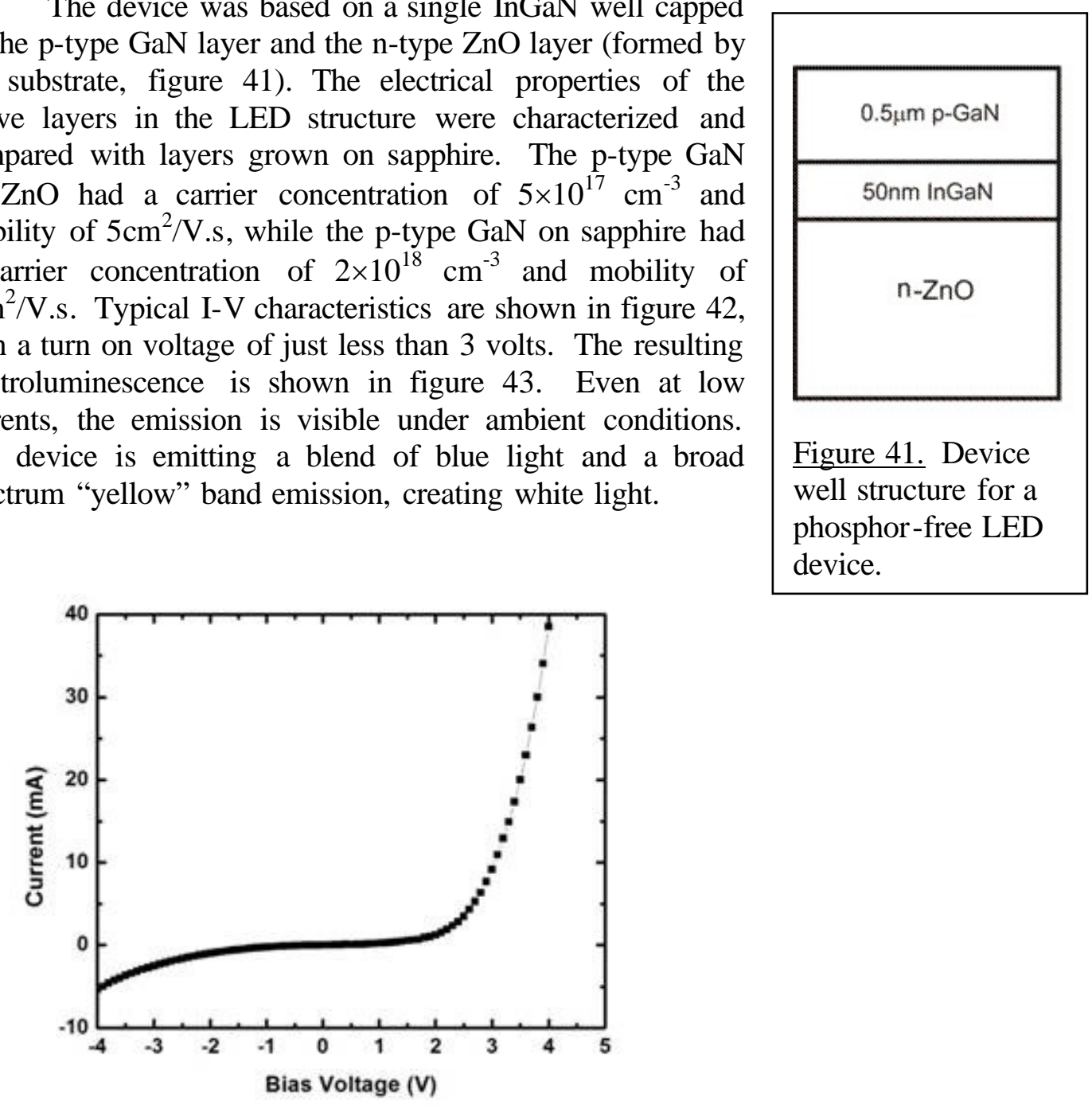

Figure 42. I-V characteristics of a prototype phosphor free white LEDs. 


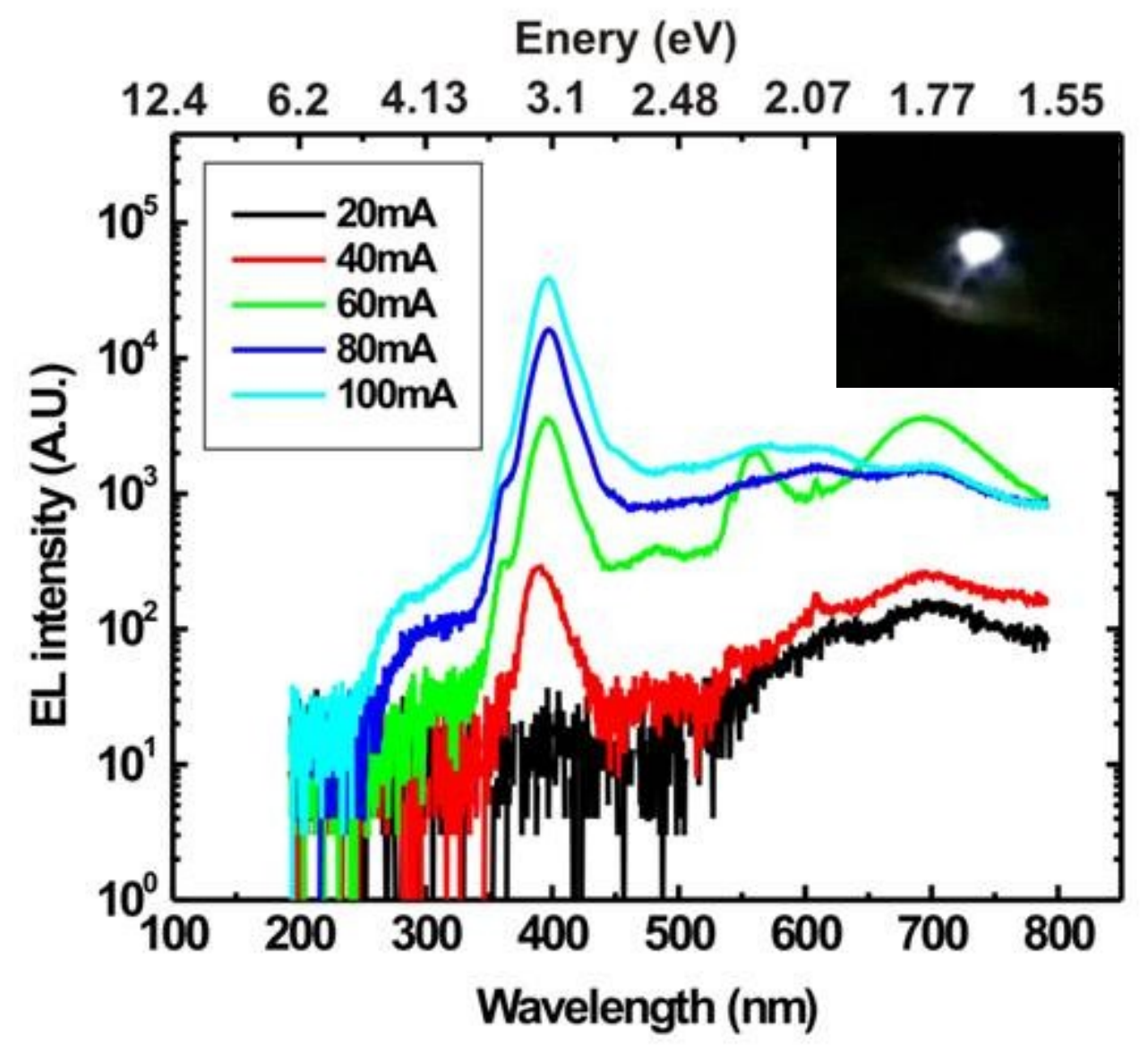

Figure 43. Spectral and visible (inset) emission from phosphor free white LEDs.

It is estimated that the efficiency of the device is less than 20 lumens per watt. However, the prototype device structures where based on a single quantum well structure with a well thickness of $50 \mathrm{~nm}$. This is greater than 10 times the well thickness of commercial LEDs. The thicker wells were used for two reasons. First, the InGaN served not only as an active layer, but also as a structural layer to provide a smooth surface on which to grow the p-type GaN. Therefore, thicker InGaN was chosen. It is anticipated that typical well thicknesses of $2-5 \mathrm{~nm}$ would provide devices that are much more intense and efficienct.

\section{CONCLUSIONS AND FUTURE WORK}

This program was extremely successful. We can conclude the following:

- The growth of doped $\mathrm{ZnO}$ crystals with excellent structural and optical properties. 
- Production of lattice-matched InGaN on $\mathrm{ZnO}$ on a commercial platform.

- Production of phosphor-free white LEDs grown on ZnO.

For this technology has substantial promise for commencial application. For this to happen, two critical technical hurdles must be overcome. The first is the elimination of diffusion of $\mathrm{Zn} / \mathrm{O}$ into the nitride active layers. This could be overcome in several ways. The first is the incorporation of diffusion barrier layers into the device structures. The second is the growth of thicker lattice matched buffer layers prior to the growth of the LED structure. The second technical hurdle is a reduction in the surface roughness of the lattice matched InGaN on $\mathrm{ZnO}$. By engineering the surface of the InGaN on $\mathrm{ZnO}$ layer, thinner quantum wells could be used in the device design shown in figure 41. Once the roughness was reduced to a level that permits the growth of a well of $2-5 \mathrm{~nm}$ thick, the simple device design would provide more lumens of output with a higher efficiency than that obtained in this program.

\section{References :}

1. F. Hamdani, M.Y., D. Smith, H. Tang, W. Kim, A. Salvador, A. Botchkarev, J. Gibson, A. Polyakov, M. Skowronski, and H. Morkoc, Microstructure and optical properties of epitaxial GaN on $\mathrm{ZnO}$ (0001) grown by reactive molecular beam epitaxy. Joumal of Applied Physics, 1998. 83(2): p. 983.

2. E. Hellman, D.B., D. Wiesmann, and I. Brener, Growth of Ga-face and N-face GaN films using ZnO Substrates. MRS Intemet Journal of Nitride Semiconductor Research, 1996. 1.

3. X. Gu, M.R., A. Teke, D. Johnstone, H. Morkoc, B. Nemeth, and J. Nause, GaN epitaxy on thermally treated c-plane bulk $\mathrm{ZnO}$ substrates with $\mathrm{O}$ and $\mathrm{Zn}$ faces. Applied Physics Letters, 2004. 84(13): p. 2268.

4. T. Deguchi, K.S., A. Nakamura, T. Sota, R. Mattsuo, S. Chichibu, and S. Nakamura, Quantum-Confined Stark Effect in an AlGaN/GaN/AlGaN Single Quantum Well Structure. Japanese Joumal of Applied Physics, 1999. 38: p. L914.

5. T. Ohgaki, S.S., N. Ohashi, I. Sakaguchi, and et. al., Structure and properties of GaN films grown on single crystalline ZnO substrates by molecular beam epitaxy. Journal of Crystal Growth, 2005. 275: p. e1143.

6. A. Kobayashi, J.O., and H. Fujioka, J. Appl. Phys. , 2006. 99(123513 ).

7. G. Namkoong, S.B., K. Lee, E. Trybus, W. Doolittle, and et. al., III-nitrides on oxygen- and zinc-face Zn) substrates. Applied Physics Letters, 2005. 87(18): p. 184104.

8. Pankove, J.I. and e. al., Appl. Phys. Lett. , 1974. 24(281).

9. Jacob, G. and e. al., J. Cryst. Growth 1977. 42(136).

10. Bergman, P. and e. al., J. Appl. Phys. , 1987. 61(4589).

11. Popovici, G. and e. al., Appl. Phys. Lett. , 1997. 71(23 ). 
12. Suzuki, T., et al., Relation between interdiffusion and polarity for MBE growth of GaN epilayers on ZnO substrates. Current Applied Physics, 2004. 4(6): p. 643.

13. Lagerstedt, O. and e. al., J. Appl. Lett. , 1974. 45(2266).

14. Monemar, B. and e. al., J. Appl. Lett. , 1980. 51(625).

15. Monemar, B. and e. al., Physica B 2006. 376(440).

16. A. Kobayashi, Y.S., K. Miyamura, J. Ohta, and H. Fujioka, Structural properties of GaN grown on Zn-face $\mathrm{ZnO}$ at room temperature. Joumal of Crystal Growth, 2007. 305: p. 70.

17. H. K. Cho, J.Y.L., K. S. Kim, and G. M. Yang, J. of Crystal Growth, 2002. 197(203).

18. Paszkiewicz, R., et al., MOVPE GaN Grown on Alternative Substrates. Crystal Research and Technology, 2001. 36: p. 971.

19. Li, N., et al., Growth of GaN on ZnO for Solid State Lighting Applications. Proceedings of SPIE, 2006. 6337: p. 63370Z.

20. Kobayashi, A., et al., Room Temperature Layer by Layer Growth of GaN on Atomically Flat ZnO. Japanese Joumal of Applied Physics, 2004. 43: p. L53.

21. Kobayashi, A., J. Ohta, and H. Fujioka, Low temperature epitaxial growth of $\mathrm{In}_{0.25} \mathrm{Ga}_{0.75} \mathrm{~N}$ on lattice-matched $\mathrm{ZnO}$ by pulsed laser deposition. Joumal of Applied Physics, 2006. 99: p. 123513.

22. D. Doppalapudi, S.N.B., K. F. Ludwig Jr., and T. D. Moustakas, J. Appl. Phys., 1998. 84(1389).

23. Ho, I. and G.B. Stringfellow, Appl. Phys. Lett., 1996. 69(2701).

24. N. A. EI-Masry, E.L.P., S. X. Liu, and S. M. Bedair, Appl. Phys. Lett., 1998. 72(40).

25. Z. C. Feng, T.R.Y., R. Liu, and T. S. A. Wee, Mater. Sci. in Semi. Proce. , 2002. 5(39).

26. Karpov, S.Y., MRS Intemet J. Nitride Semicond. Res., 1998. 3(16).

27. A. Tabata, L.K.T., L. M. R. Scolfaro, J. R. Leite, A. Kharchenko, T. Frey, D. J. and D.S. As, K.Lischka, J. Furthmüller, and F. Bechstedt, Appl. Phys. Lett., 2002. 80(769).

28. C. A. Parker, J.C.R., S. M. Bedair, M. J. Reed, S. X. Liu, and N. A. El-Masry, and L.H. Robins, Appl. Phys. Lett., 1999. 75(2566).

29. M. Smith, G.D.C., J. Y. Lin, H. X. Jiang, M. Asif Khan, Q. Chen, Appl. Phys. Lett., 1996. 69(2837).

30. K. L. Teo, J.S.C., P. Y. Yu, E. R. Weber, M. F. Li, W. Liu, K. Uchida, H. and N.A. Tokunaga, K. Matsumoto, Appl. Phys. Lett., 1998. 73(1697).

31. Zunger, A., Handbook of Crystal Growth, ed. D.T.J. Hurle. Vol. 3. 1994, New York: Elsevier. p.998.

32. I. H. Lee, I.H.C., C. R. Lee, E. J. Shin, D. Kim, S. K. Noh, S. J. Son, K. Y. Lim, and H. J. Lee, J. Appl. Phys., 1998. 83(5787).

33. P. Cantu, F.W., P. Waltereit, S. Keller, A. E. Romanov, U. K. Mishra, S. P. DenBaars, and J. S. Speck, Appl. Phys. Lett. , 2003. 83(674).

34. M. K. Chena, Y.C.C., J. Y. Chen, C. M. Wub, C. C. Yang, K. J. Mad, J. R. Yange, and A. Rosenauerf, J. Crystal growth, 2005. 279(55). 
